\title{
Summaries of FY 1997 Engineering Research
}

September 1998

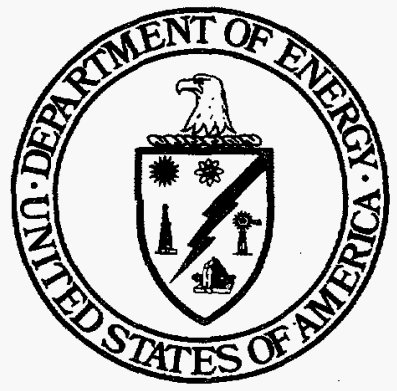

\section{U.S. Department of Energy}

Office of Energy Research

Office of Basic Energy Sciences

Division of Engineering and Geosciences

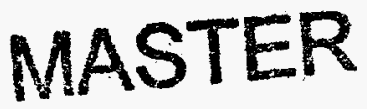
Washington, DC 20585 


\section{DISCLAIMER}

This report was prepared as an account of work sponsored by an agency of the United States Government. Neither the United States Government nor any agency thereof, nor any of their employees, makes any warranty, express or implied, or assumes any legal liability or responsibility for the accuracy, completeness, or usefulness of any information, apparatus, product, or process disclosed, or represents that its use would not infringe privately owned rights. Reference herein to any specific commercial product, process, or service by trade name, trademark, manufacturer, or otherwise does not necessarily constitute or imply its endorsement, recommendation, or favoring by the United States Government or any agency thereof. The views and opinions of authors expressed herein do not necessarily state or reflect those of the United States Government or any agency thereof. 


\section{DISCLAIMER}

Portions of this document may be illegible in electronic image products. Images are produced from the best available original document. 


\section{Foreword}

This report documents the Basic Energy Sciences (BES) Engineering Research Program for fiscal year 1997; it provides a summary for each of the program projects in addition to a brief program overview. The report is intended to provide staff of Congressional committees, other executive departments, and other DOE offices with substantive program information so as to facilitate governmental overview and coordination of Federal research programs. Of equal importance, its availability facilitates communication of program information to interested research engineers and scientists. Each BES Division administers basic, mission oriented research programs in the area indicated by its title. The BES Engineering Research Program is one such program; it is administered by the Engineering and Geosciences Division of BES. Dr. Robert E. Price is technical manager of the Engineering Research Program; inquiries concerning the program may be addressed to him, in writing, by phone at (301) $903-5822 /(301)$ 903-3565 or by fax at (301) 903-0271 (additional information and updates are accessible on World Wide Web, http://er.doe.gov).

In preparing this report we asked the principal investigators to submit summaries for their projects that were specifically applicable to fiscal year 1997. The summaries received have been edited if necessary, but the press for timely publication made it impractical to have the investigators review and approve the revised summaries prior to publication. For more information about a given project, it is suggested that the investigators be contacted directly. 


\section{Introduction}

The individual project summaries follow the program overview. The summaries are ordered alphabetically by name of institution; the table of contents lists all the institutions at which projects were sponsored in fiscal year 1997.

Each project entry begins with an institutional-departmental heading. The names of investigators are listed immediately below the title. The funding level for fiscal year 1997 appears to the right of address. The summary description of the project completes the entry. A separate index of Principal Investigators includes phone number, fax number and e-mail address, where available. 


\section{Program Review BES Engineering Research}

The BES Engineering Research Program is one of the component research programs which collectively constitute the DOE Basic Energy Sciences Program. The DOE Basic Energy Sciences program supports energy related research in the physical and biological sciences, and in engineering. The chief purpose of the DOE Basic Energy Sciences Program is to provide the fundamental scientific base on which identification and development of future, national energy options will depend. The major product of the program becomes part of the body of data and knowledge upon which the applied energy technologies are founded; the product is knowledge relevant to energy exploration, production, conversion and use.

The BES Engineering Research Program was started in 1979 to help resolve the numerous serious engineering issues arising from efforts to meet U.S. energy needs. The program supports fundamental research on broad, generic topics in energy related engineering topics not as narrowly scoped as those addressed by the shorter term engineering research projects sponsored by the various DOE technology programs. Special emphasis is placed on projects which, if successfully concluded, will benefit more than one energy technology. During the first year several workshops were sponsored for the purpose of identifying energy related engineering research needs and initial priorities. Representatives from industry, academic institutions, national laboratories, and leading members of professional organizations (Engineering Societies Commission of Energy, American Society of Mechanical Engineers, Society of Automotive Engineers, and Joint Automation and Control Committee) participated in the workshops. In addition to the participants in the workshops, staff representatives from the DOE technology programs and other leading U.S. energy engineering experts made significant contributions to the setting of program priorities. There resulted from this process a strong confirmation of the need for a long range, fundamental engineering research program with two major goals. The broad goals that were established by this process for the BES Engineering Research Program are:

1) To extend the body of knowledge underlying current engineering practice so as to create new options for enhancing energy savings and production, for prolonging useful equipment life, and for reducing costs without degradation of industrial production and performance quality; and

2) To broaden the technical and conceptual base for solving future engineering problems in the energy technologies. 
In this process, it was further established that to achieve these goals, the BES Engineering Research Program should address the following topics identified as essential to the progress of many energy technologies:

1) Advanced Industrial Technology: improvement of energy conversion and utilization, opening new technological possibilities, and improvement of energy systems.

2) Fluid Dynamics and Thermal Processes: broadening of understanding of heat transfer in nonsteady flows, methodology for reducing vibrations and noise in heat exchangers, and engineering aspects of combustion.

3) Solid Mechanics: continuum mechanics, fracture mechanics, thermomechanical behavior in severe environments, aging and lifetime reliability of structures.

4) Dynamics and Control of Processes and Systems: development and use of information describing system behavior (system models), performance criteria, and theories of control optimization to achieve the best possible system performance subject to known constraints.

A Scoping Workshop held in December 1985 confirmed the continued needs for research in these topical areas. Because of budgetary limitations, the implemented BES Engineering Research Program is somewhat less broad than the program envisioned above. At present, equal emphasis is being placed in three carefully selected, high priority research areas; namely,

1) Mechanical Sciences including fluid mechanics heat transfer, and solid mechanics, but excluding purely computational efforts.

2) System Sciences including process control, instrumentation, and intelligent systems.

3) Engineering Analysis including nonlinear dynamics, data bases for thermophysical properties of fluids, modeling of combustion processes for engineering application, and foundations of bioprocessing of fuels and energy related wastes.

These areas contain the most critical elements of the four topics enumerated above; as such they are of importance to energy technologies both in the short and long term, and therefore of immediate programmatic interest. It should be noted that other areas of basic research important to engineering are monitored elsewhere in BES. For instance, separation sciences and research on thermophysical properties are among the responsibilities of the Chemical Sciences Division, while microscopic aspects of fracture mechanics are in the domain of the Material Sciences Division. As resources permit, other high priority areas are being added to the Engineering Research 
Program. Thus, as a result of previous growth in the program budget an important development took place in the Engineering Research Program: two major concentrations of research were initiated.

First, a new program was organized at Oak Ridge National Laboratory dealing with intelligent machines in an unstructured environment. Some resources are available for coordinated, more narrowly focussed, related, high quality research at universities and other research centers. All such activities are supported and administered directly by the Engineering Research Program, but some coordination of efforts with the ORNL program may prove useful.

Secondly in FY 1985, a collaborative research effort was started between MIT and Idaho National Engineering Laboratory. The collaboration is in two distinct areas: Automated Welding, and Fracture Mechanics. Collateral, high quality research efforts at other institutions, including Plasma Process Engineering are supported by the Engineering Research Program.

In the expectation of a future modest growth of this Program, three International Workshops on Two Phase Flow Fundamental were held one in September 1985 and the other in March 1987. The meetings were used to identify basic research needs in the field of two phase flow and heat transfer; summary reports of the workshops are available from the Program Office. The proceedings of the two workshops have been published as volumes in the series "Advances in Heat and Mass Transfer" (Hemisphere Publishing Company). A third international workshop held in June 1992 surveyed the status of the field. The proceedings have been published by $\mathrm{CRC}$ Publishing Company, also in the series "Advances in Heat and Mass Transfer".

Two additional workshops were held during 1988 . The first dealt with possible research opportunities in the field of novel devices using the new high temperature superconductors. The second addressed research needs for bioprocessing of fuels and energy related wastes. Reports of both workshops have been published. Additional funds had been provided in FY 1992 to initiate research in the above mentioned bioprocessing area. Of interest are relevant studies at the intersection of biology, biochemistry, and chemical engineering.

Another workshop aimed at identifying research opportunities to mitigate the effects of aging in energy production and distribution systems took place in October i992. The proceedings have appeared in Applied Mechanics Reviews.

During the past year, workshops have been planned to examine engineering research in the areas of micro- and nano-meter systems. It is expected to result in an increase in research in micro- and nano-meter systems. 
Research projects sponsored by the BES Engineering Research Program are currently underway at universities, private sector laboratories, and DOE national laboratories. In fiscal year 1997 the program operating funds available for research amounted to about $\$ 15.3$ million. The distribution of these funds among various institutions and by topical area is illustrated on the next page. Project funding levels are mostly in the range of $\$ 50,000$ to $\$ 150,000$ per year. Typical duration of a project is three to four years, with some projects expected to last as long as ten years or more. The BES Engineering Research projects stem almost without exception from competitive grant applications. Applications which anticipate definite results in less than two years are usually referred to the appropriate DOE technology program for consideration. All those interested in submitting a proposal are encouraged to discuss their ideas with the technical program manager prior to submission of a formal proposal. Such discussion helps to establish whether or not a potential project has a reasonable chance of being funded. The primary considerations for possible support are the technical quality of the proposal and the professional standing of the principal investigators and staff. An effort is made to attract first rate, younger research engineers and energy oriented applied scientists. A high technical caliber of research is maintained by requiring that the projects supported have potential for a significant contribution to energy related engineering science, or for an initial contribution to a new energy relevant technology. Sponsored projects are selected primarily for their relevance to DOE mission requirements; the contribution to energy related graduate education is an important consideration. Thus projects sponsored at universities are essentially limited to advanced theoretical and experimental studies usually performed by faculty members, staff research scientists, and doctoral candidates. 

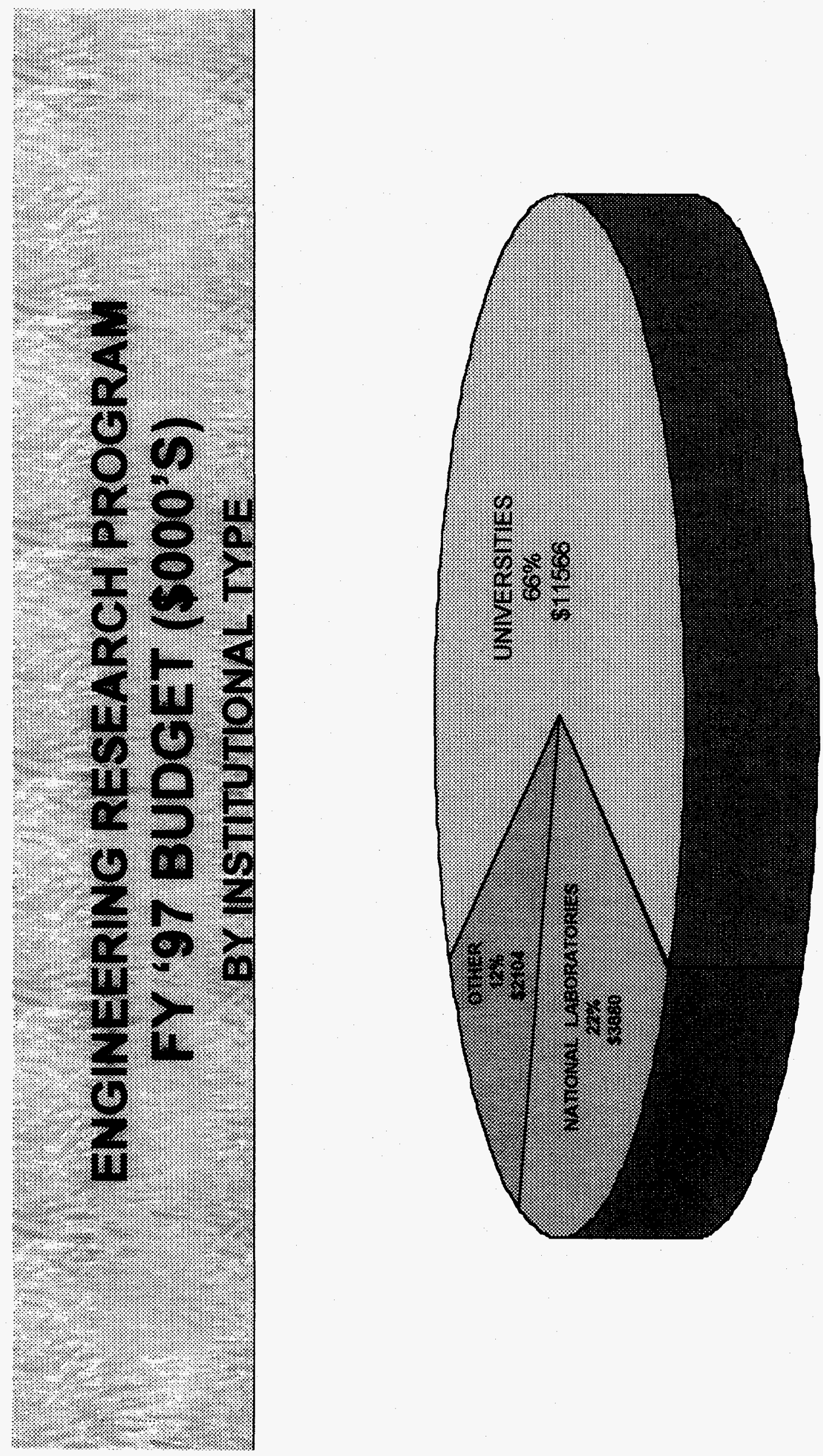


\section{Table of Contents}

University of Alabama . . . . . . . . . . . . . . . . . 1

University of Arizona $\ldots \ldots \ldots \ldots \ldots \ldots \ldots \ldots \ldots \ldots$

Battelle Memorial Institute $\ldots \ldots \ldots \ldots \ldots \ldots \ldots \ldots 2$

Brown University . . . . . . . . . . . . . . . . . 3

University Of California, Berkeley .................. 3

University Of California, Los Angeles . . . . . . . . . . . . 6

University Of California, San Diego . . . . . . . . . . . . 7

University Of California, Santa Barbara . . . . . . . . . . . 12

California Institute Of Technology $\ldots \ldots \ldots \ldots \ldots \ldots \ldots 13$

Carnegie Mellon University $\ldots \ldots \ldots \ldots \ldots \ldots \ldots \ldots . \ldots \ldots$

University Of Chicago . . . . . . . . . . . . . . . . . . . . . 14

Clarkson University ...................... 15

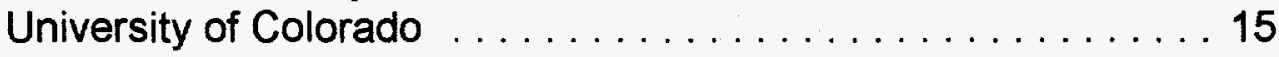

Cornell University . . . . . . . . . . . . . . . . . . . . . . . 16

Dartmouth College .......................... 17

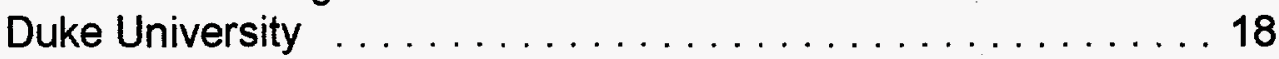

Florida State University . . . . . . . . . . . . . . . . . . 18

Georgia Institute of Technology . . . . . . . . . . . . . 19

Idaho National Engineering and Environmental Laboratory . . . . 19

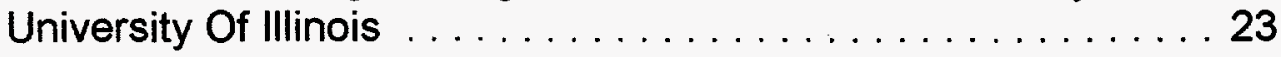

Johns Hopkins University . . . . . . . . . . . . . . . . . 26

Robert H. Kraichnan, Inc. . . . . . . . . . . . . . . . . . 26

Lawrence Berkeley National Laboratory . . . . . . . . . . . . . . . . 27

Lawrence Livermore National Laboratory . . . . . . . . . . . . . 27

The Lovelace Institutes . . . . . . . . . . . . . . . . . . . . . 28

McDermott Technology, Inc . . . . . . . . . . . . . . . . 29

University Of Maryland . . . . . . . . . . . . . . . . . . 29

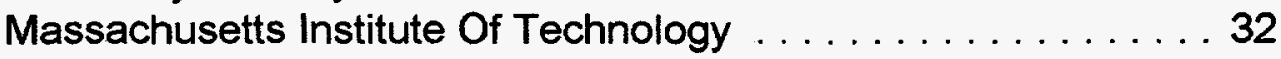

University Of Minnesota . . . . . . . . . . . . . . . . 36

National Academy of Sciences/National Academy of Eng . . . . 37

National Aeronautics and Space Administration . . . . . . . 39

National Center for Manufacturing Sciences . . . . . . . . . . . 39

National Institute Of Standards And Technology ......... 40

The City University Of New York, The City College . . . . . . . . 41

State University of New York . . . . . . . . . . . . . . . . . 43

Northwestern University . . . . . . . . . . . . . . . . . . . . . . . . 44

University Of Notre Dame . . . . . . . . . . . . . . . . . . . . . . 47

Oak Ridge National Laboratory . . . . . . . . . . . . . 47 


\section{Table of Contents (continued)}

Oregon State University $\ldots \ldots \ldots \ldots \ldots \ldots \ldots$

Pennsylvania State University . . . . . . . . . . . . . . . 49

Princeton University . . . . . . . . . . . . . . . . . . . . 49

Purdue University . . . . . . . . . . . . . . . . . . . . . . . . . 50

Rensselaer Polytechnic Institute . . . . . . . . . . . . . . . 51

Rice University . . . . . . . . . . . . . . . . . . . . 53

University Of Rochester . . . . . . . . . . . . . . . 53

Rockefeller University . . . . . . . . . . . . . . . . . . . . . . . . . 54

Sandia National Laboratories . . . . . . . . . . . . . . . . . . . . 55

Science Applications IntI Corp . . . . . . . . . . . . . . . . 55

Stanford University . . . . . . . . . . . . . . . . . . . . . 56

University Of Texas At Austin . . . . . . . . . . . . . . . . . . . 59

Tufts University . . . . . . . . . . . . . . . . . . . . . . . 60

University of Washington . . . . . . . . . . . . . . 61

Washington University . . . . . . . . . . . . . . . . . 62

Washington State University $\ldots \ldots \ldots \ldots \ldots 6 . \ldots \ldots 6$

University Of Wisconsin . . . . . . . . . . . . . . . . 66 63 


\section{University Of Alabama}

Dept of Mathematics

Tuscaloosa, AL 35487

\section{Hydrodynamic Instabilities and Coherent Structures}

A. Frenkel

The objective of this research is to further the fundamental understanding of stability properties of several far-from- equilibrium fluid systems which are relevant to energy engineering sciences. In particular, flows periodic in space and possibly in time are studied as models to gain insights into such turbulence phenomena as large-scale coherent structures, eddy viscosity, and the inverse cascade of energy. Film flows--such as core-annular ones--are important to, e.g., lubricated pipelining of viscous oils. The large-scale evolution equations for the different systems may exhibit common features, such as pattern formation and coherent structures.

Some of the results are as follows: A rigorous iterative method was suggested for the problems of periodic-flow stability. The possibility of negative isotropic large-eddy viscosity was demonstrated, resolving a rather long-outstanding question. A weakly nonlinear Landau-type theory was constructed for an intermediate-scale instability of a periodic flow. The spatial structure of the saturated disturbances of this non-uniform flow is significantly different from previously known, uniform cases.

For film flows, a perturbative method capable of yielding both the evolution descriptions and the parametric conditions of their validity was suggested. ighly nonlinear evolution equation of a wavy flow down a cylinder was obtained. Its numerical simulations yielded an excellent agreement with experiments. They also revealed, for the first time ever in numerical simulations, irreversible coalescences of soliton-shaped coherent structures; these are now thought to play an important role in the wave dynamics of film flows. Also, a theory of a flow down an inclined plane was constructed. Simulations of the evolution equation showed a remarkable agreement with three-dimensional wave patterns observed in recent experiments.

Some fundamental questions concerning commonly used perturbative approaches were clarified. Certain deficiencies of well-known evolution equations were pointed out.

\section{University of Arizona}

Aerospace \& Mechanical Eng

Tucson, AZ 85721

\section{Film Cooling in a Pulsating Stream}

I. Wygnanski, H. Fasel, A. Ortega

Wall jets have traditionally been used for boundary layer control and in heat transfer applications such as film-cooling and evaporation enhancement. This summary presents the status of an investigation that attempts to understand the complexities behind momentum and energy transport in externally excited wall jet flows. The approach combines experimentation, direct numerical simulations (DNS) and stability theory. The experimental results for the laminar wall jet

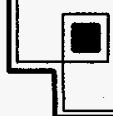

Engineering Research 
flowing over a constant temperature surface substantiated the stability theory for both the streamwise velocity and temperature fields for low excitation levels. For higher forcing levels, the experimental findings were compared to incompressible DNS which solve the incompressible Navier Stokes equations in vorticity-velocity formulation in conjunction with the energy equation. Both experiments and DNS revealed that forcing at high excitation levels causes a significant reduction in the skin friction and a concomitant increase in heat flux relative to the unforced case. A significant growth of the sub-harmonic component was also observed within the measurement domain. It is believed that these large scale and low frequency structures appear to contribute to the reduction and augmentation of the shear stress and heat flux. Understanding the complexities of the turbulent wall jet with heat transfer is currently the focus of the research.

\section{Battelle Memorial Institute}

Engineering Mechanics Dept

Columbus, OH 43201-2693

\section{An Investigation of History-Dependent Damage In Time-Dependent Fracture Mechanics} F. Brust, Jr.

In order to meet the demand imposed by future technology, new plants with increased energy efficiency must operate at relatively high temperatures. Additionally, the existing power generation equipment in the United States continues to age and is being used far beyond its intended life. Some recent failures have clearly demonstrated that the current methods for insuring safety and reliability of high temperature equipment is inadequate. Owing to these concerns, a thorough understanding of high temperature failure initiation and propagation in materials exposed to variable mechanical and thermal loading is very important.

In the past, the evolution of damage has been addressed through a macroscopic theoretical model (developed as part of this effort) which attempt to predict the crack growth and failure response of material components exposed to high temperature conditions. However, micromechanical processes such as diffusion of atomic flux into grain boundaries, elastic accommodation and creep deformation of the material and grain boundary sliding do contribute significantly to the nucleation and growth of voids leading to failure. Understanding gained by consideration of micro-mechanics of cavity growth is crucial for developing damage-based constitutive models as well as methodologies for life prediction of structural components. While the application of this understanding in estimating life of structural materials experiencing high temperature creep has met with some success, it is of limited use for structural components experiencing complex load histories under high temperature conditions.

A micro-mechanical model accounting for rate-controlling microscopic processes has been developed as part of this effort. To date, both sustained and variable load histories have been investigated in two-dimensional geometries. The results illustrate the importance of accounting for nonlinear changes in geometry, grain-boundary diffusion processes, elastic accommodation of the surrounding material as well as more realistic constitutive laws for creep deformation. Current efforts involve investigating different load histories and three-dimensional effects. In addition, the ultimate goal of this effort is to establish a firm connection between the micro- and macro-mechanical models thereby leading to the development of appropriate methodology for life prediction of structural components exposed to high temperature conditions involving complex load histories.

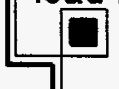

Engineering Research 
Brown University

Dept of Chemical Engineering

Providence, RI 02912

$\$ 99,860$

Simulation and Analysis of Dyanmic Failure of Ductile Materials

L.B. Freund

A central goal in the mechanics of materials is the determination of parameters which characterize macroscopic failure of materials in terms quantifiable characteristics of their microstructure. The motivation is to establish which characteristics account for macroscopic failure, with a view toward improvement of failure resistance through material selection or microstructural design. In the present project, emphasis is on the behavior of ductile structural alloys under high rate loading conditions. Thus, the dominant mechanism of plastic deformation is crystallographic slip and material strength degrades through nucleation, growth and coalescence of micro-voids. Plastic strains in such processes can be large and strain localization is common. The approach is to adapt methodologies for analysis of elastic-viscoplastic systems to problems selected on the basis of their relevance to safety of pressure vessel and piping systems, to materials processing and metal forming technologies, and to structural reliability under dynamic loading. Initial emphasis has been on failure of an explosively loaded ring expanding under plane strain conditions, a configurationwhich has been studied experimentally. Calculations reveal that strain localization sites, or necks, are more pervasive under rapid loading, and the spacing of necks decreases with increase in loading rate. The influence of inertia on bifurcation of deformation states is also being investigated theoretically. The project is being carried out in collaboration with colleagues involved in experimental research on dynamic ductile failure at the California Institute of Technology.

\section{University Of California/B}

Dept of Chemical Engineering

Berkeley, CA 94720

\section{Enzyme Adsorption and Activity at Liquid-Liquid Interfaces H. Blanch}

Protein adsorption at oil/water interfaces is the primary phenomenon governing formation of stabilized protein adsorption, and the kinetics of interfacially active enzymes. We apply pendant drop tensiometry, total internal reflection fluorescence spectroscopy (TIRFS) and thin film balance/interferometry to study enzyme adsorption behavior. We have also constructed a reactor to follow enzyme kinetics at oil/water interfaces. Pendant drop tensiometry has been used to follow the dynamic interfacial tension of several well characterized proteins at the heptane interface and hydroxynitrile lyase from Prunus amygdalus at the interface of several different oils. Transient interfacial tension studies show that at short times (seconds), predicted diffusional effects are observed. At extended times (hours), adsorbed proteins form gel-like layers. Adsorption is accompanied by conformational changes and interactions between adjacent molecules, often resulting in the formation of a quasi-solid macroscopic skin at the interface. As the nature of the oil is changed from nonpolar to more polar the surface pressure increases demonstrating higher adsorption and greater enzyme denaturation. We have observed that the increased denaturation correlates more closely with oil/water interfacial tensions than with

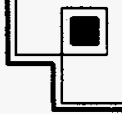


solubility's. The effect of oil on enzyme interfacial activity will be examined using the reactor described above. We also use fluorescence spectroscopy to follow protein adsorption and to gain information on protein molecular conformation in the adsorbed state. A novel TIRFS for liquid/liquid interfaces has been developed in our lab. The apparatus enables the dynamics of protein adsorption to be followed while also giving us a means to test the reversibility of surface reactions in a non-invasive manner. Studies have shown a fluorescein-beta-casein conjugate irreversibly adsorbs at the oil/water interface after long term exposure. At early times we have observed exchange between native, adsorbed b-casein and bulk fluorescein-beta-casein conjugate. The ability to exchange decreases with continued exposure of the adsorbed protein to the interface. Native and thermally denatured proteins have been studied using the thin film balance. The denatured protein stabilized a thicker film than the native protein at a given concentration demonstrating the expanded conformation of the denatured protein.

\section{University Of California/B}

Dept of Mechanical Engineering

Berkeley, CA 94720

\section{Ultrashort Laser Heating and Phase Change in Liquids C.-L. Tien, C. P. Grigoropoulos}

The general objective of this research program is to achieve a better understanding of laser-liquid interactions from the thermal perspective. Two main tasks were proposed for investigation: the fundamental mechanisms of high-intensity, short-pulse laser heating of liquids and pulsed-laser-induced vapor-phase nucleation and growth. This report presents the progress of the research for these two tasks.

\section{Mechanisms of High-Intensity Laser Heating of Liquids}

Residual moisture is a problem in microelectronics and microelectromechanical systems (MEMS) devices which can result in corrosion, device degradation, and even complete device failure. Traditional methods of removing liquids are often not viable due to constraints on the maximum allowable temperature or may not remove all of the liquid. In addition to thermal heating, ultrashort-pulse lasers can remove undesirable surface species through an electronic desorption mechanism. When the laser pulse duration is shorter than the relaxation time of the material, typically on the order of 1 picosecond (10-12 s), non-equilibrium heating of the energy carriers and the lattice results making electronic desorption possible.

Electronic desorption from metal and semiconductor surfaces due to short-pulse laser heating has been analytically and computationally modeled. For metal substrates, regimes of thermal and electronic desorption were identified along with characteristic length and time scales. A feasibility experiment demonstrating the recovery of microstructures which stuck to the underlying substrate due to stiction was conducted. Stiction is the permanent adhesion of one part of a microstructure to another and the capillary forces generated by the drying rinse liquids play a major role in its occurrence.

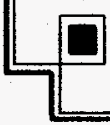




\section{Pulsed Laser-Induced Phase-Change and Ablation in Absorbing Liquids}

The phase-change process and the generation of acoustic transients in the pulsed laser-induced ablation of absorbing liquids were investigated [1]. Aqueous solutions having different absorption coefficients were irradiated by a nanosecond pulsed laser. The ablation process including the vapor cavity formation and the acoustic-wave propagation were visualized by laser flash photography. The generated pressure transients were measured using a broadband piezoelectric pressure transducer. Simultaneous optical transmission detection was also carried out in order to elucidate the vapor-phase kinetics and determine the ablation threshold. The effects of the liquid absorption on the ablation mechanism and the acoustic-pulse generation were investigated. The results showed that the ablation is initiated by cavitation driven by the tensile component of the produced thermoelastic stress. The ablation threshold corresponds to peak surface temperatures that are substantially below the saturation temperature at the ambient pressure. Three distinct stages were identified in the ablation process: the initial stage with negligible surface deformation and with ejection of a small amount of vapor, the second stage with violent vapor plume and bulk liquid jet ejection that is accompanied by significant surface deformation, and the final stage of large scale hydrodynamic motion. The pressure transient is generated thermoelastically at low laser fluences, but the contribution of the recoil momentum exerted by the ablation plume becomes dominant at high laser fluences.

The interaction of pulsed laser radiation of hundreds of microseconds duration with bulk liquids was examined. For these slower heat input rates, the phase-change process occurs thermally. In the free boundary case, the ablation threshold is marked at peak temperatures near the saturation temperature. A surface tension driven hydrodynamic instability inhibits the growth of the vapor phase, leading to cavity collapse and generation of acoustic pressure in the liquid. In the presence of a rigid boundary, the violent collapse of the formed bubble is accompanied by the release of a strong pressure pulse into the liquid.

\section{Pulsed-Laser-Induced Vapor-Phase Nucleation and Growth}

Irradiation of a KrF excimer laser beam of nanosecond pulse duration on an absorbing solid surface immersed in a transparent liquid induces rapid thermal expansion and explosive vaporization of the liquid accompanied by pressure pulse generation. The pressure contribution due to the thermal expansion of the solid sample and bubble formation was measured by the probe beam deflection method as well as by a broadband piezoelectric transducer. The pressure generation is enhanced by the bubble expansion in the superheated water for laser fluences exceeding the bubble nucleation threshold. The bubble growth dynamics was examined by a novel, non-contact optical interference technique. This work quantified, for the first time, the transient behavior and growth rate of a submicron thick bubble layer in the nanosecond time scale. Experiments are now focused on the study of pulsed laser-induced cavitation and phasechange in absorbing liquids. 


\section{University Of California/LA}

Mech, Aero \& Nuclear Eng Dept

$\$ 140,712$

School of Eng \& Applied Science

Los Angeles, CA 90024-1597

\section{Basic Studies of Transport Processes in Porous Media \\ l. Catton}

The objective of this project is to develop an understanding of the governing physical processes at a level appropriate for development of macroscopic models for use in the analysis of engineered energy systems. Current work considers both single-and two-phase flows. Efforts are both theoretical (numerical in some cases) and experimental. Emphasis is placed upon the development of mathematical models for single-and two-phase turbulent transport in highly porous media with regular, non-uniform, isotropic and non-isotropic characteristics. The basis of the work is a hierarchical heterogeneous volume averaging theory (VAT) using fully turbulent models with Reynolds stresses and fluxes in every pore space building from the lowest level. Boundary and interface conditions are incorporated at various scales leading to descriptions of momentum, heat and mass transport in porous media.

The VAT equations for developed flow and diffusive processes in a random, highly porous medium have been developed along with the statistical and numerical methodology needed to treat the fluctuation terms for various assigned random porous morphologies. Models have been developed to describe turbulent flow, diffusion of admixtures, and energy processes in highly porous media. Using second order turbulent models, equation sets have been obtained for laminar and turbulent filtration and two-temperature or two-concentration diffusion in non-isotropic porous media and interface exchange and micro-roughness. The equations differ from those found in the literature. We continue to work on closure problems that arise in their implementation.

In the search for closure it was shown that terms in the stationary integro-differential heat-and mass transport equations based on VAT that are normally ignored, must be kept if the medium morphology characteristics are spatially dependent. It was also found that polymorphism of the near surface fluid layers in fine pores cause differences in the bulk liquid viscosity leading to inapplicability of the Darcy law.

The transport equations needed to model fluid-media interface pressure and heat resistance losses and the heterogeneity of the medium morphology have been developed to correct pressure loss experimental results separating the bulk pressure drop from the inlet and outlet losses. A method of normalization of data from various porous media measurements was developed from the VAT based equations and shown collapse many of the correlations developed from the Reynolds-Forchheimer type of equation.

Diode Array Velocimetry (DAV) and a new method of data analysis have been used to obtain the turbulent characteristics of flow in a pipe and they compare well to the results of other methods. This method will be used to study the impact of various regular surfaces on the turbulent structure of the flow to obtain data needed for closure of the VAT based equations. Experimental measurements of the Fanning friction factor for foamed ceramics and metals are nearing completion. Heat transfer measurements will soon begin.

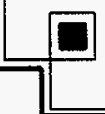

Engineering Research 
University Of California/LA

Physics Dept

Los Angeles, CA 90024

$\$ 77,000$

\section{Nonlinear Waves in Continuous Media: Application to Stochasticity and Energy Concentration \\ S. Putterman}

We propose to study the effects of nonlinear interactions on the fate of energy that is injected into a continuous medium so as to drive it far off-equilibrium. In some cases nonlinear processes lead to a spectacular concentration of energy such as is apparent in sonoluminescence; the transduction of sound into light. This phenomenon will be studied with the goal of understanding the light emitting mechanism and the underlying bifurcations which control its parameter space. An attempt will be made to define and locate a parallel phenomena in crack motion and triboelectrification. Nonlinearity can also lead to the randomization of energy as evidenced by the stochastic properties of turbulence. In this direction we propose to study turbulently interacting capillary waves. We will investigate collective modes of turbulence and the development of correlations on ultra-long time scales. Finally, with regard to the appearance of structure in nonlinear systems, we will search for higher dimensional solitons. These structures resist diffraction as well as dispersion.

\section{University of California/SD}

Scripps Inst of Oceanography

$\$ 75,000$

La Jolla, CA 92093-0402

\section{Broadband Signals: Signal Processing in Chaos H. Abarbanel}

The analysis of chaotic signals observed in measurements on physical systems is of importance in energy problems ranging from fluidized bed flows in fossil energy applications to determination of the natural climate variability to the uncovering of simple models for complex behavior in fluid flows. This research has developed tools for this analysis which allows one to reconstruct the multivariate state space of a system from observations, time lagged, of a single dynamical variable. The time delay, the dimension, and properties of the strange attractor can all be determined from these data. The concept of unfolding the attractor using the method of global false nearest neighbors and then determining locally the number of dynamical degrees of freedom using the local version of this has brought to the study of complex behavior a robustness which allows it to be used in engineering analysis and design. The algorithms developed for this purpose have also been used in a variety of applications requiring the separation of a chaotic signal from another information bearing signal or 'noise'. The tool kit of these algorithms is being ported to a common interface for use in the energy related sciences.

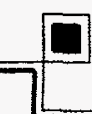




\section{University Of California/SD}

Dept of Chemistry, 0340

La Jolla, CA 92093

\section{Noisy Nonlinear Systems}

\section{K. Lindenberg}

The broad objective of this project is to investigate the interplay of nonlinear deterministic dynamics with spatial and temporal fluctuations.

Work has continued on coupled anharmonicnonlinear arrays. In soft anharmonic classical arrays at finite temperatures theentropy-driven energy localization mechanism called stochastic localization has been probed by comparing the transition rates of bistable impurities embedded in a chain of soft oscillators with those in a harmonic chain. The differences can be dramatic. In quantum systems at zero temperature it is shown that ubiquitous semiclassical models are not consistent with the behavior obtained for the stationary states of the system via new and highly accurate variational results.

The directed motion of a particle in a periodic non-symmetric ratchet potential driven by symmetric time-correlated forces has been investigated. An expression for the induced current at large correlation times that correctly captures the qualitative features of the ratchet motion has been derived. Color-induced current reversal of a rigid dimer moving in a ratchet has been demonstrated numerically.

The escape rate of a Brownian particle over a potential barrier is strongly affected by barrier fluctuations. Such a system can exhibit a maximum in the escape rate as a function of the barrier fluctuation parameters. An expression for the escape rate that shows this resonant activation has been derived and has been confirmed quantitatively via numerical simulations.

\section{University Of California/SD}

Inst for Nonlinear Science, 0402

La Jolla, CA 92093

\section{Structure and Modeling of Turbulence E. Novikov}

Well developed turbulent flows are encountered in many energy engineering enterprises, as well as in environmental, aerospace and naval engineering. The number of degrees of freedom for such flows is enormous and direct numerical simulations (DNS) are impossible now and in a foreseeable future.

One of the major goals of this project is to advance the knowledge of the structure of turbulence, aiming it at a subgrid-scale modeling for the large-eddy simulations (LES) and at a transformation from homogeneous to nonhomogeneous turbulent flows. The developed approach for achievement of this goal includes: conditional averaging of the Navier-Stokes equations (NSE); use of Markov processes with dependent increments (consistent with NSE); breakdown coefficients (for a description of intermittency) and associated infinitely divisible probability distributions. 
Among the obtained results are: probability distribution for the three-dimensional (3D) vectors of velocity increments, which has an unusual form and experimentaiiy observed exponential asymptotics; a new scaling for experimentally and numerically observed 3D "vortex strings", 2D coherent vortices and coherent structures of scalar fields; the whole 3D conditionally averaged vorticity field (with fixed vorticity in one point) and its characteristics, obtained by DNS on the CM-5 parallel computer, using finite differences on $(256)^{3}$ grid; statistical characteristics of microcirculations in 3D and vorticity increments in 2D; an imbedding of the phenomena of intermittency and scale similarity into the theory of infinitely divisible probability distributions; probability distributions and moments of the breakdown coeffcients for the energy dissipation, compared with experimental data for the Taylor-scale Reynolds number 3200; a hierarchy of subgrid- scale models, tested by DNS and by LES for isotropic and free-surface turbulence; an analytical transformation from homogeneous to nonhomogeneous turbulent flows, tested by data for free-surface turbulence in a presence of a solid boundary; a Markovian body force for a modeling of solid boundary; distribution of droplets in turbulent spray.

\section{University Of California/SD}

Inst for Nonlinear Science

La Jolla, CA 92093

\section{Spatiotemporal Wave Patterns: Models and Analysis}

\section{Rabinovich, L. Tsimring}

Key phenomena of pattern evolution and spatio-temporal disorder in nonequilibrium media and complex systems are investigated experimentally and theoretically. We consider the dynamics of defects against the background of regular two-dimensional patterns (rolls, hexagons, quasi-crystalline structures); the self-organization of an irregular ensemble of defects and the formation of domain walls, as well as the interaction of two-dimensional patterns with large-scale flows. Experiments are carried out with Faraday ripples, Benard-Marangoni convection, and an oscillating soap film. The experiments with soap film demonstrate that for a given steady flexural mode pattern, increasing the amplitude of mode oscillation leads to the excitation of the planar vortices. For large enough amplitude in a square cell, one can see the spatio-temporal chaos. We proposed a theoretical model based on the wave-boundary interaction of excited Marangoni waves. The model reveals a vorticity generation mechanism in vibrating soap films. The model qualitatively reproduces many steady vortex patterns observed in the experiment.

Spatio-temporal patterns in lattice models of the cortex are also addressed. We proposed a nonlinear dynamical model that is able to translate the temporal information coded by temporal patterns of spike's sequences into the neuronal activity of distributed processing system and to store this information. As a result of external stimulation the unstable spatio-homogeneous mode of the model evolves into the quasistationary mode with complex spatiotemporal dynamics.

We also demonstrated that spatiotemporal patterns from one excitable media can be conveyed through a small number or channels into another equivalent excitable media.

We proposed a theoretical model for pattern formation in a thin granular layer on a vibrating plate. This model, in agreement with experiments, exhibits subcritical transition, rolls, squares, and localized stated - oscillons.

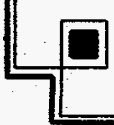

Engineering Research 


\section{University Of California/SD}

Dept of Physics, 0319

$\$ 126,731$

La Jolla, CA 92093

\section{Experimental Study of 2D Traveling-Wave Patterns in Binary Fluid Convection \\ C. Surko}

This research involves the study of convection in fluid mixtures of ethanol and water. This system has proven to be a good model for studying nonequilibrium traveling-wave phenomena, and it also provides insights into other behavior in doubly diffusive systems in which the transport (in this case, of heat and concentration) occurs on different time scales. As a fluid dynamical system, the phenomena of interest are closely related to atmospheric and oceanographic flows. As a model for traveling-wave dynamics, it provides useful insights in understanding physical systems of practical importance, such as mode control in large-aspect-ratio lasers.

One focus of current work is the study of 2-D traveling wave patterns in a large-aspect-ratio container. In an effort to characterize these patterns and their evolution, numerical techniques were developed to identify the phase defects and to calculate their trajectories. The statistics of the defects provides an accurate measure of the disorder in thepatterns. Work in progress explores the extent to which the motion of the defects can be predicted and used to determine the evolution of the patterns.

Recently, an instability was discovered when the fluid layer is heated from above -- a situation which is stable in a pure fluid. A large-wavenumber pattern develops which appears to be closely related to salt fingering in thermohaline convection. The observed flow patterns exhibit many interesting characteristics, including a wave number spectrum which evolves in time and persistent stochastic dynamics.

\section{University Of California/SD}

Institute of Nonlinear Science

$\$ 104,030$

La Jolla, CA 92093-0402

\section{Modeling of Process Control}

\section{Tsimring}

Traditionally, for ease of operation and analysis, technological systems (manufacturing systems, communication systems, etc.) have been designed to behave in as linear a manner as possible. Increasingly tight specifications, environmental considerations and economic pressures are pushing the operational windows into regions where assumptions of linearity tend to break down. This project aims to the development of tools for building nonlinear models for complex temporal and spatiotemporal systems. These models can be used for system classification, control, and inter-instrument validation. Our models are based on input/output data and do not require the knowledge of underlying dynamical equations. Building on this modeling we devised several control schemes for nonlinear chaotic systems. 
One way to control a system is to synchronize it with a known system or a dynamical model. Our recent work included building models for generalized synchronizatiori between non-identical systems and developing numerical and experimental tools for detecting it.

We studied the effects of noisy and band-limited channels between coupled chaotic systems on the quality of synchronization and communication.

We studied numerically and experimentally a new data-based entrainment technique for trajectory planning of an unknown nonlinear system. This technique allows one to build an input-output model directly from data and drive the system towards the desired trajectory (not necessarily periodic) using an accessible control parameter.

\section{University Of California/SD}

Dept of App Mech \& Eng Sci

$\$ 146,307$

La Jolla, CA 92093-0411

\section{Fundamental Studies of Spray Combustion}

F. Williams, P. Libby, S. C. Li

This research, involving both experimental and theoretical components, has addressed droplet and spray characteristics in counterflowing streams. A test rig which permits a wide variety of investigations including nonpremixed, premixed and partially premixed systems in both laminar and tubulent streams represents the main experimental setup. A two-component phase Doppler particle analyzer (PDPA) is employed to obtain droplet size, number density and two components of velocity. In addition, in portions of spray flames not containing droplets, measurements are made of the profiles of temperature and of concentrations of stable species, including the oxides of nitrogen. Thermocouples and gas choromotography and a nitrogen-oxides analyzer are used for the measurements. One recent research effort has been concentrated on the reduction in NOx formation in two-stage flames by water droplets or carbon dioxide. A paper from this research was presented by S.C. Li at AMSE Turbo Expo '96, June 10-13, 1996, Birmingham, UK and it was selected the 'Best Technical Paper' by International Gas Turbine Institute. A second effort has been devoted to staged combustion of methanol and other liquid fuels; in this case reactants are partially premixed to improve the characteristics of spray combustion such as reducing emissions of oxides of nitrogen and extending the ranges of stable spray combustion. Three papers from our research on spray combustion have been accepted for publication, including one invited review paper entitled 'Spray Stagnation Flames' in Progress in Energy and Combustion Science. One paper, entitled 'NOx Reduction in Diesel-Fuel Flames by Addition of Water and $\mathrm{CO}_{2}$ ' will be presented at 1997 Diesel Engine Emissions Reduction Workshop at UCSD sponsored by DOE. Experimental work on spray turbulent combustion is underway. 


\section{University Of California/SB}

Dept of Physics

Santa Barbara, CA 93106

\section{Bifurcations and Patterns in Nonlinear Dissipative Systems}

\section{G. Ahlers, D. Cannell}

This project consists of experimental investigations of heat transport, pattern formation, and bifurcation phenomena in non-linear non-equilibrium fluid-mechanical systems. These issues are studies in Rayleigh-Benard convection, using both pure and multicomponent fluids. They are of fundamental scientific interest, but also play an important role in engineering, materials science, ecology, meteorology, geophysics, and astrophysics. For instance, various forms of convection are important in such diverse phenomena as crystal growth from a melt with or without impurities, energy production in solar ponds, flow in the earth's mantle, geo-thermal stratifications, and various oceanographic and atmospheric phenomena. Our work utilizes computer-enhanced shadowgraph imaging of flow patterns and high-resolution heat transport measurements.

We studied convection in a gas $\left(\mathrm{CO}_{2}\right)$ under pressure (about 30 bar) with a Prandtl number Pr=1, in a very large aspect ratio sample (radius/height up to 150). For parameter values where time-independent parallel straight rolls were theoretically predicted to be stable, we found a state of spiral-defect chaos (SDC). These experimental results have stimulated a great deal of theoretical work, using both analytical and numerical methods and various model equations for convection.

We extended our experiments on SDC to small values of Pr by using mixtures of gases. If one component of the mixture has a large and the other a small molecular mass, values of Pr as small as 0.14 can be reached. The first appearance of SDC shifts closer to the onset of convection as Pr decreases. This gives information about the origin of SDC, but has not yet been explained quantitatively.

We investigated convection in a nematic liquid crystal in a horizontal magnetic field $(H)$. We found excelent agreement with recent theoretical predictions for the bifurcation line $R-C(H)$, and for the convection-roll orientations as a function of $\mathrm{H}$. We also used this system to study convection when two phases (the nematic and isotropic phases) are present in the cell. Convection in the presence of a first-order phase change is relevant to convection in the earth's mantle; ours are the first quantitative experiments relevant to this important problem.

\section{University Of California/SB}

Dept of Chemical \& Nuc Eng

Santa Barbara, CA 93106

\section{Wave Turbulence Interactions}

\section{S. Banerjee}

The work is part of an ongoing research effort aimed at understanding transport processes across a gas-liquid interface. The research includes both experimental and computational studies focusing on the effects of waves on turbulence in a liquid stream. Two- and three-dimensional

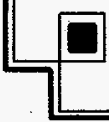

Engineering Research 
mechanically generated waves, spanning the range of conditions from sinusoidal waves to micro-breaking are studied. Experimental work involves using a 3-D LDA synchronized with ultra-sonic surface position measurements. Flow visualization with micro-bubbles and high speed video are used for direct examination of flow structures. DPIV technique are further applied to the flow visualization video images for in-depth quantitative flow field analysis.

Experimental results indicate that the turbulence intensity away from the wall was substantially increased as a result of the waves, especially close to the interface, which suggests possible enhancement of interface scalar transport. The increase appears to be a consequence of turbulence production in the outer flow region due to wave-induced Reynolds stress. Turbulence intensity in the wall region and turbulence wall structures were not significantly affected. Wave-induced turbulence was also seen in a laminar flow by flow visualization.

Direct numerical simulation of gas and liquid flowing across a mobile interface is being conducted. The results will allow more detailed examination of the effects of waves on turbulence production.

\section{California Institute of Technology}

Graduate Aeronautical Laboratories

$\$ 99,904$

Pasadena, CA 91125

\section{Dynamic Failure Characterization of Ductile Steels \\ A. Rosakis}

The goal of the current research program is to study dynamic crack initiation in ductile steels (304 Stainless Steel and A 533 B Steel) at different loading rates and to establish appropriate dynamic fracture criteria. A variety of infrared and visible optical methods and high speed photography are used in this study. As part of our work we have developed novel experimental methods to achieve our tasks.

The precracked steel specimens are subjected to dynamic three-point bend loading by impacting them in a drop weight tower. This impact results in deformation and fracture initiation. During the dynamic deformation and fracture initiation process the time history of the transient temperature in the vicinity of the crack tip is recorded experimentally using a high-speed infrared detector. The dynamic temperature trace in conjunction with the HRR solution is used to determine the time history of the dynamic J-integral, $\mathcal{J}^{d}(t)$, and to establish the dynamic fracture initiation toughness, $\mathcal{J}\left(t_{0}\right)=\mathcal{J}_{\text {crit }}$, where $t=t_{0}$ is the time of fracture initiation. The measurements made using highspeed thermography are validated through comparison with determination of $\boldsymbol{J}^{d}(t)$ by dynamic optical measurements of the crack tip opening displacement. Finally, the micromechanisms of fracture initiation are investigated by studying the fracture surface using scanning electron microscopy. 
Carnegie Mellon University

Chemical Eng Dept

Pittsburgh, PA 15213

$\$ 170,000$

Systematic Process Synthesis and Design Methods for Cost Effective Waste

Minimization

\section{Biegler, I. Grossmann, A. Westerberg}

This project is developing a novel integrated approach for process synthesis and design to address recent environmental challenges. The approach provides rigorous trade-offs among raw material and energy costs, capital investment and waste treatment. Issues of waste minimization addressed include:

1. Economic design of reactor networks that minimize conversion of raw materials to waste byproducts.

2. Synthesis of separation sequences to isolate, redirect and recycle byproducts.

3. Integrated synthesis and design of reaction, separation and energy systems with environmental and operability concerns, also dealing with process uncertainty.

Reactor designs that are optimized and synthesized simultaneously with the process flowsheet can have the greatest impact for process improvement, both from an environmental and an economic perspective. A quantitative targeting approach for reactor networks is permitting the reduction of waste byproducts at the source. The second task concentrates on the synthesis of flexible separation processes (displaying azeotropic and liquid/liquid behavior) that can accomodate variations in the process. Cooperative search methods are being developed to overcome the combinatorial explosion of alternatives. The third task combines structural optimization and problem decomposition at various modeling levels in order to screen alternatives based on economic, environmental and operabilty trade-offs. Novel decomposition methods are being developed for the synthesis and operation of utility plants that must cope with changes in demands and waste treatment requirements.

\section{University Of Chicago}

The Enrico Fermi Institute

Chicago, IL 60637

$\$ 177,633$

\section{Fundamentals and Techniques of Nonimaging Optics \\ R. Winston}

Nonimaging optics departs from the methods of traditional optical design to develop instead techniques for maximizing the collecting power of concentrating elements and systems. Designs which exceed the concentration attainable with focusing techniques by factors of four or more and approach the theoretical limit are possible. Our theoretical work on nonimaging designs has led to our group experimentally demonstrating ultra-high flux from sunlight which exceeds previous results by substantial factors. Our "tailored edge ray" method of nonimaging design has advantages for many applications both in energy collection and in illumination. Progress on

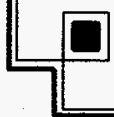

Engineering Research 
non-rotationally symmetrical designs has led to potentially important advances in illumination systems. Our work on generalized radiance and instrument functions has contributed to a better understanding of the foundations of radiometry.

\section{Clarkson University}

Dept of Chemical Engineering

$\$ 143,423$

Potsdam, NY 13699-5705

\section{Surfactant Effects on Mass Transfer for Bubbles at Large Reynolds Numbers J. McLaughlin, R. J. Nunge}

The main objective of this project is to develop a better understanding of mass transfer between bubbles and impure liquids. New techniques will be developed that can be used to analyze mass transfer in a broad range of problems. The focus is on bubbles between $0.5 \mathrm{~mm}$ and $2.0 \mathrm{~mm}$ in water. Issues of particular interest are the transient period during which surfactants adsorb onto the bubble and the effect of the transient on bubble dissolution.

The scientific approach involves laboratory experiments and numerical simulations. Experiments will be done to measure the velocity and dissolution of bubbles in both pure water and water containing controlled amounts of surfactants. The numerical simulations will provide information about the flow and concentration fields around the bubble and the dependence of the transient period on surfactant sorption kinetics. A new numerical technique will be used to simulate mass transfer between a bubble and the surrounding liquid.

Computer codes for the solution of the Navier-Stokes and mass transfer equations have been developed and mass transfer results have been obtained for Peclet numbers of $O(102)$. A Monte Carlo code for larger Peclet numbers is being developed. The equipment for the experiments has been constructed.

\section{University of Colorado}

Dept of Mechanical Engineering

Boulder, CO 80309-0427

\section{Electromechanical Behavior and Properties of High-T $\mathrm{T}_{c}$ Superconducting Tapes}

\section{S. Datta}

The objective of this research program is to develop a fundamental understanding of the electromechanical behavior of high- $T_{c}$ superconducting tapes, and to rigorously establish the connection between thermomechanical response and transport properties (critical fields and currents). We are motivated by the recent progress that has been made in developing longlength, finite-width Bi-based tapes for industrial applications at both liquid nitrogen and liquid helium temperatures, and the recognition that thermomechanical phenomena (stresses, cracking, damage) during both processing and service have significant impact on the realizable critical currents of these tapes. The research focuses on modeling the complicated three-dimensional<smiles>C/C=C\c1ccccc1</smiles> 
stress state in superconducting tapes when subjected to thermal and multiaxial mechanical loading. Features of the materials that complicate analysis include the strong anisotropy of the oxide superconductor, the inelastic constitutive behavior of the stabilizing metal, and the temperature dependent material properties of both the superconductor and metal phases. Our studies will provide a fundamental understanding of thermomechanical behavior and failure mechanisms. They will quantify the influence of controllable parameters such as layer thickness and interface toughness, and will identify strategies to tailor mechanical failure modes to optimize electrical performance. We will correlate our analyses with critical current measurements to determine the intrinsic and extrinsic effects of stress on superconducting properties. We will also study, both theoretically and experimentally, the propagation of ultrasonic waves in the superconducting tapes with the aim of providing valuable mechanical property characterization. The ultrasonic study will specifically address the effect of the finite width of the tape on elastic guided waves and will be closely coordinated with the laser ultrasonic study at Idaho National Engineering and Environmental Laboratories by $\mathrm{Dr}$. K. Telschow. While our study focuses on primarily on high- $T_{\mathrm{c}}$ superconducting tapes, the theoretical and experimental framework is quite broad, having numerous other applications including the analysis of multilayer actuators, multichip modules in electronic packaging, coated and graded materials, and failure of bonded or welded parts.

\section{Cornell University}

Mechanical \& Aerospace Eng $\quad \$ 89,469$ Ithaca, NY 14853

Nonlinear Dynamics and Control of Large Arrays of Coupled Oscillators: Application to Fluid-Elastic Problems

\section{$F$. Moon}

The goal of this study is to investigate the development of low dimensional models for fluid-elastic phenomena. In the Cornell research we have focused on the flow of air around a row of flexible circular cylinders which is typical in heat-exchanger systems. In the past year we have obtained both theoretical and experimental results. These results concern the nature of the bifurcation or critical flow velocity at which the tubes loose stability and also on the nature of the unstable mode of coupled-tube vibrations. In summary we have observed the following:

I) we have experimental evidence for a sub critical Hopf bifurcation;

ii) the critical speed for bifurcation decreases as the number of flexible cylinders increases;

iii) the unstable vibration mode has the nature of coupled helical wave solutions;

iv) a theoretical model has been developed, based in part on measured fluid-elastic constants, which shows both the decrease in critical flow velocity with number of flexible tubes and shows the helical wave solutions.

The current year's effort is directed toward pursuing the nature of the nonlinear terms in the theoretical mode responsible for the sub critical Hopf bifurcation that has been observed in experiments.

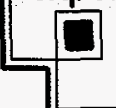

Engineering Research 
In a separate study, we have also explored controlling chaos in mechanical systems with impact and friction. These experiments are modeled after the theoretical work of ütt, Grebogi, and Yorke at University of Maryland and the experiments of Hunt at Ohio University. We have designed and implemented a circuit and actuator that can suppress chaos in an impact oscillator as well as one with dry friction. This result may be relevant to control of fluid-elastic tube vibrations under impact.

\section{Cornell University}

Sibley School of Mech \& Aero Eng

$\$ 141,131$

Ithaca, NY 14853

\section{Experiments on Velocity and Scalar Fields in High Reynolds Number Laboratory Flows Z. Warhaft}

Turbulence commonly occurs in chemical reactions, combustion, mixing, pollution and flow around vehicles (causing high drag). In many of these problems progress is hindered because of our ignorance of turbulence structure and dynamics. By means of fundamental experiments we attempt to shed light on basic turbulence structure, in particular at the small scales where it is thought that the turbulence behavior becomes universal, i.e., it is independent of the particular way it has been generated.

In order to do these studies, highly energetic turbulence must be generated so that the small scale structure can be achieved. We have recently developed active grids that can produce turbulence at (Taylor-scale) Reynolds numbers close to 1000; much higher than that achievable using the very largest computers (both now and for the foreseeable future). We have just completed studies of scalar mixing, and the effects of Reynolds number variation in flows with and without shear. In particular we have found incipient small scale anisotropy in both the scalar and velocity fields. This suggests that turbulence models will have to be more subtle in dealing with small scale structure and we will have to modify our ideas on the physics of the mixing processes in high Reynolds number turbulent flows.

\section{Dartmouth College}

Thayer School of Engineering

Hanover, NH 03755

$\$ 127,684$

\section{Two-Phase Potential Flow \\ G. Wallis}

The objective is to develop results for well-defined situations that will serve as a basis for evaluating or developing specific terms, particularly those involving fluid inertia, in basic models of two-phase flow. During the last year emphasis has been on the "drift force" on a particle in an inviscid flow with vorticity. Basic theory, experiments and CFD simulations complement each other and substantially agree. Experiments, analysis and CFD simulations are also being used to study transients in fluidized particulate systems, particularly those involving slugs, shock waves and expansion waves.

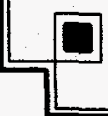

Engineering Research 


\section{Duke University}

Dept of Physics

Durham, NC 27706

\section{Experimental and Analytical Investigations of Flows in Porous Media R. Behringer, G. Johnson, J. Georgiadis}

Research Objectives: The objectives of this project include the characterization, both theoretical and experimental of fluid flows through porous media. The particular focus is on fluid convective flow in porous media. Convection in porous media occurs, for instance, when a layer of fluid-saturated porous media is heated from below.

The characterization of porous convections includes both pure fluids and mixtures of different fluids. Long term benefits will be an improvement in the theortical and practical applications of flow in porous media in the presence of temperature gradients. Relevant practical situations include petroleum and hydrology problems where heating occurs.

Scientific Approach: The primary approach is experimental, and involves the use of Magnetic Resonance Imaging (MRI) as a powerful new tool to see the flow inside of porous media. The project involves the use of a MRI apparatus and a precision thermal apparatus for carrying out the convection experiments. Additional theoretical work now includes an analysis of the convective model equations with the goal of understanding recent experimental results. These results show a strong coupling between the solid structure of porous media and the flow states.

Current Status of Project: Recent work has been described in Shattuck et al. Phys. Rev. 75, 1934 (1995). These experiments show the strong coupling between pore space structure and flow patterns. An additional longer paper is in progress. This work forms the basis of Mark Shattuck's thesis. Continuing work will focus on experimental MRI studies of binary mixture convection in porous media.

\section{Florida State University}

Supercomputer Computations

$\$ 106,090$

Research Institute

Tallahassee, FL 32306

\section{Theoretical and Computational Studies of Pattern Formation J. Viñals}

We seek to improve our understanding of the mechanisms underlying the formation of complex spatio-temporal patterns in systems that are driven outside of thermodynamic equilibrium, to characterize the macroscopic properties of such states, and to apply the methodology to situations of interest in Materials Science, materials processing and Engineering. Two avenues of research are being pursued: Patterns and chaos in fluids, and an order parameter formulation of two-phase fluids. In the former, we utilize amplitude and order parameter equations to describe the formation of patterns, their instabilities and the transition to chaotic states in extended systems. In the latter, we introduce and order parameter formulation to describe moving boundary problems in two phase fluids, and consider microstructure evolution in systems not readily tractable with conventional hydrodynamics.

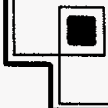


Georgia Institute of Technology

Computational Mechanics Center

Atlanta, GA 30332

$\$ 88,993$

Residual Life and Strength Prediction and Life-Enhancement, of Structural in Aging Energy Production Systems

S. Atluri

The objective of this reseach project is to study the structural integrity and damage tolerance of aging energy systems. In collaboration with the Fracture Mechanics Group at the University of Washington, hybrid experimental-numerical analyses are being carried out, to establish an energy-like parameter to characterize elastic-plastic and creep fracture that may occur in high temperature pressure vessels. The $T^{*}$ integral parameter is being developed as a candidate parameter. Using :(i) analytical solutions for linear elastic infinite domains containing line or planar cracks, the faces of which are subjected to arbitrary tractions, (ii) the finite element method to model the uncracked structure, and (iii) the generalized radial return algorithms for plasticity and creep, a cost-efficient and accurate Inelastic Finite Element Alternating Method is being developed. Elastic-plastic analyses of stable tearing in 2- and 3-D situations, using the IFEAM are being carried out. Finally, strategies for life extension of energy systems, using composite repairs are being studied.

\section{Idaho National Engineering and Environmental Laboratory}

Energy Res \& Applications Dept

Idaho Falls, ID 83415

\section{Fundamentals of Thermal Plasma Processes}

J. Fincke

This project is of a coordinated experimental-theoretical research project on thermal plasma processing of materials. This work is primarily focused on the development of advanced diagnostic and computational techniques and their application to obtain a better and more detailed understanding of the fundamental physical and chemical processes occurring in nonequilibrium thermal plasmas with entrained particles. The techniques thus developed and the information and insights they provide, can then be directly applied to process design, optimization, and scale-up. The diagnostic and computational techniques already developed under this project now represent the state of the art in this area. During the next five years of this project, we propose to further extend and generalize these techniques to permit their application to several additional topics of timely importance in the thermal plasma processing of materials, namely (1) functionally gradient materials (FGMs), (2) reactive plasma spraying and (3) plasma chemical synthesis of nanophase materials. These topics share some common features and physics which make it efficient and cost-effective to consider them together. They form a natural progression and will be pursued sequentially in the above order, but with significant overlap. 
Idaho National Engineering and Environmental Laboratory Materials Science Division

Idaho Falls, ID 83415

\section{Application of Intelligent Control Systems to Mixed-Culture Bioprocesses J. Johnson, D. Stoner}

Even though the control of biologically-based processes is an extremely complex, multi-task activty, conventional control systems operate at the most rudimentary of levels. That is, standard set point control and alarm systems are used to monitor and maintain process parameters, while process performance and recovery from upset conditions are dependent on the operator's judgment and experience. Artificial intelligence has the potential of incorporating a human's experience and rationale for the supervision of a bioprocess into a computer controlled system, thus, radically changing the manner in which bioprocesses are supervised and controlled. The challenges that confront the development of intelligent systems for bioprocessing control are due to the limitations of the intelligent technologies; the manner in which these technologies may be integrated into a control system; and the limitations inherent in the mathematical models that are used to describe microbial metabolism and activity.

The overall goal of this program is to demonstrate that the productivity of bioprocesses can be improved by integrating intelligent systems with conventional bioprocess control technologies. In addition, the project will demonstrate the application of intelligent control to a complex system that no analytic model can adequately describe. Investigators at the Idaho National Engineering Laboratory have designed, built and evaluated hierarchical hardware and software systems for the intelligent control of continuous flow, stirred tank reactors. The control system was developed and evaluated using Thiobacillus ferrooxidans, a microorganism for which there is a moderate amount information. This learning-based control system was successfully applied to an uncharacterized culture of iron-oxidizing thermophilic microorganisms.

The hierarchical control systems had, as their lower level platform, an integrated system of set point control, data acquisition, data analysis and equipment diagnostics programs. The control system used on-line sensors and off-line measurements to determine the state of the system. A two-level control system determined what actions were required to meet process goals. These actions were carried out by computer controlled actuators, including pumps, gas-flow valves, a heater, and a stirrer. The upper level of the control system, the supervisory control system, used stochastic learning to determine what the system parameters, e.g., $\mathrm{pH}$, dilution rate, and temperature, should be, based on the state of the system. Lower level fuzzy systems and standard classical methods implemented the controllers that operated the system. A diagnostic system analyzed the sensor data for inconsistencies and provided a log of the system operation. 


\section{Idaho National Engineering and Environmental Laboratory}

Materials Technology Group

$\$ 369,000$

Idaho Falls, ID 83415-2218

\section{Elastic-Plastic Fracture Analysis Emphasis on Surface Flaws W. Reuter, J. Epstein, W. Lloyd}

The objective is to improve design and analytical techniques for predicting the integrity of flawed structural components. Tests are being conducted on materials ranging from linear elastic to fully plastic. The latter extends beyond the range of a J-controlled field. Specimens containing surface cracks are used to simulate the fracture process (crack growth initiation, subcritical growth, and catastrophic failure) that may occur in structural components.

Metallography and microtopography techniques have been developed to measure crack tip opening displacement and crack tip opening angle for comparison with analytical models. Moire interferometry techniques are used to evaluate and quantify the deformation in the crack region. These studies have resulted in the ability to predict crack growth initiation of specimens containing surface cracks using constraint and fracture toughness data obtained from standard fracture toughness specimens. Results are being transferred to industry in the form of an ASTM Test Standard on Surface Cracked Specimens (Structures) that is presently being developed.

Due to the complexity of studying the fracture process in weldments, diffusion bonded specimens were used initially to simulate a weldment. This provided an opportunity to study the fracture process in a model weldment of either a butt weld or a single " $V$ " groove geometry that contained neither a heat affected zone nor residual stresses. This work has been completed. Two weldments have been fabricated using commercial ship building technology for a single " $V$ " groove with one having matched weld metal and the second an overmatched weld metal. Another weldment has been fabricated using a one-half " $K$ " weld joint design Characterization of the microstructure and of local tensile properties is presently in progress. Testing of fracture toughness specimens, specimens containing surface cracks, and modified specimen geometries has been initiated.

\section{Idaho National Engineering and Environmental Laboratory} Materials Technology Group $\$ 508,000$ Idaho Falls, ID 83415

\section{Intelligent Control of Thermal Processes}

H. B. Smartt, J. A. Johnson

This project addresses intelligent control of thermal processes as applied to gas metal arc welding. Intelligent control is defined as the combined application of process modeling, sensing, artificial intelligence, and control theory to process control. The intent of intelligent control is to produce a good product without relying on post-process inspection and statistical quality control procedures, by integrating knowledge of process engineering practice and process physics with real time weld quality and process state sensing into control algorithms. The gas metal arc welding process is used as a model system; considerable fundamental information on the process has been developed at INEL and MIT. Research is being conducted on analytical modeling to couple nonlinear aspects of molten metal droplet formation and transfer with base 
metal melting and resultant weld pool geometry, and integration of knowledge-based control methods (including artificial neural networks and fuzzy logic based connectionist systems) with iterative learning control methods. Results are being transferred to industrial partners through a related DOE-EE CRADA on Intelligent Diagnostics, Sensing, and Control of Thin Section Welding, and a work-for-others CRADA to an industrial end user.

The focus of present work is specifically on develoment of real time optimal parameter selection for pulsed current gas metal arc welding of steel and aluminum structures. This work is being conducted in collaboration with a related DOE-BES welding fracture mechanics program being conducted at INEEL and MIT and is expected to be of significance for the petrochemical, shipbuilding and automotive industries.

This project is part of a collaborative research program with the Massachusetts Institute of Technology.

\section{Idaho National Engineering and Environmental Laboratory Nondestructive Mat'ls Charac Grp Idaho Falls, ID 83415-2209}

\section{Coupled Mode NDE \\ K. L. Telschow}

Many engineering material applications lack microstructure characterization methods that are both applicable to the industrial environment and provide sufficient selectivity for features of interest, such as fatigue, residual stress, or specific microstructures. However, small changes in magnetization often accompany microstructural changes in industrial materials brought about by effects related to aging, lifetime reduction, and the manufacturing process that could be used as selective probes. High Temperature Superconducting Quantum Interference Devices (HTS-SQUID) technology offers the prospect for measuring these small magnetizations due to high sensitivity and wide dynamic range. This research program is seeking new selectivity in nondestructive evaluation (NDE) through local measurement of small magnetizations that arise from the dynamics of material properties brought about by electromagnetic, elastic, or optical perturbations ("coupled modes"). Selectivity comes from the material?s nonlinear interaction terms in the internal energy that can be differentiated by this coupled mode approach. Combined magnetic and ultrasonic assessments of materials in tape form and local measurement of fatigue damage in stainless steel piping are being pursued. The work builds on previous NDE research conducted at the INEEL based on noncontacting electromagnetic measurement of the superconducting properties of tapes, measurement of fatigue and aging in stainless steels, and optical ultrasonic measurement methods. 


\section{University Of Illinois}

Urbana, IL 61801

\section{Optimization-Based Robust Identification and Control of Uncertain Dynamical Systems T. Basar}

This program involves fundamental research on optimization-based robust identification and control of uncertain dynamical systems. The class of systems considered includes large scale, stochastic, nonlinear, hybrid, and distributed parameter systems, all subject to different types of static as well as dynamic uncertainties. The optimality criteria adopted include minimax, risk-sensitive, and receding horizon formulations. The main theme is optimality-based identification, control, and model simplification under severe internal and external uncertainties. Research involves not independent, but a combined design of observer/filter and control architectures, supported by optimization-based model-reduction, decomposition and aggregation techniques.

One area of current research is the derivation of optimally disturbance attenuating controllers for nonlinear systems in a receding horizon framework, when full state measurements are not available. This way an implementable controller structure can be obtained, instead of the infinite-dimensional one the original problem admits. Another topic of current study involves robust control of nonlinear systems whose dynamics are unknown, where some neural network architectures are incorporated into the design. Yet another topic is the derivation of robust controllers for some classes of uncertain infinite dimensional systems, and systems which undergo structural changes governed by a stochastic process.

\section{University Of Illinois}

Dept of Chemical Engineering

$\$ 155,000$

Urbana, IL 61801

\section{Gas-Liquid Flow in Pipelines \\ T. Hanratty}

Many operations in the petroleum industry involve the flow of gas and liquid in pipelines. Examples are offshore oil platforms and the transportation of natural gas in pipelines. The economic and safe design of these systems is handicapped because of difficulties in predicting how the phases distribute. Considerable progress has been made by relating large scale behavior to small scale interactions between the phases.

Slug flow is a pattern that exists in horizontal flows whereby slugs of highly aerated liquid flow intermittently along a pipeline. Of critical importance is the prediction when this pattern will exist and the prediction of the frequency of slugging. Usual practice is to examine the stability of a stratified flow, where the liquid flows along the bottom of a horizontal pipe and the gas concurrently above it. A different approach, which involves a study of the stability of slugs, provides an explanation of the initiation of slugging at high gas velocities and at high pressures. Measurements of the time and spatial variation of the liquid height along a pipeline is providing the physical understanding of the formation of slugs needed to predict frequency.

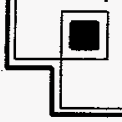

Engineering Research 
An important configuration which appears in condensers and evaporators, as well as in petroleum pipelines, is annular flow. Part of the liquid flows along the wall and part as drops entrained in the gas. A critical problem in horizontal pipes is the prediction of asymmetries due to gravity. Experimental results on these asymmetries are being used to improve our ability to improve present design methods. This work will be particularly important to understanding the performance of large diameter pipes for which the transition from stratified to annular flow occurs over a wide range of gas velocities.

\section{University Of Illinois}

Aeronautical and Astronautical

Engineering

Champaign, IL 61801

\section{Nonlinear Dynamics of Parametrically Excited Gyroscopic Systems S. Namachchivaya}

Gyroscopic systems are an important class of systems which include such problems as rotating shafts, supported pipes conveying fluid, systems subject to Lorenz forces, structures moving in fluid media, transverse vibrations of moving belts, etc. In these gyroscopic systems, there are many situations in which the system is parametrically or stochastically excited. For example, the rotating shaft may be subject to periodic or random perturbations in its applied end load or rotation rate, due to its interaction with other components. The pipe conveying fluid may be subject to similar structural excitations, as well as periodic or random fluctuations in the fluid flow rate. Such disturbances can lead to modal interactions, large amplitude oscillations, chaotic behavior, etc.

The primary goal of the research is to study the local and global dynamics of gyroscopic systems subject to periodic and random excitations, and to numerically and experimentally verify the theoretical results. The results of this research will be useful in such applications as rotating shafts, pipes conveying fluid, etc. The objectives of the research are as follows:

- In the first part of the research, the nonlinear equations of motion governing a general gyroscopic system will be systematically derived. The equations will include the effects of parametric/random excitation, symmetry-breaking, and linear and nonlinear dissipation. In addition, equations will be derived for several physical examples which are gyroscopic systems.

- In the second part of the research, the local bifurcations associated with the discretized equations of motion will be examined. Furthermore, physical applications contain inherent imperfections which break the symmetry of the problem. Such symmetry-breaking imperfections may give rise to local and global bifurcations. The effects of symmetry and the associated reduction of nonlinear equations, along with symmetry-breaking bifurcations, will also be examined. The results will then be interpreted for each of the physical applications.

- In the third part of the research, the mechanisms which give rise to global bifurcations in gyroscopic systems will be fully examined. The focus of this part is the development of general analytical techniques to predict global behavior in this class of systems. We propose to develop 
method of predicting these global phenomena in a systematic manner. In addition, the local and global behavior predicted using analytical methods will be compared with the behavior observed numerically using continuation methods, numerical integration of discretized equations of motion, etc. Once again, these results will be interpreted in light of the specific physical applications.

- In the fourth part of this research, mathematical techniques will be developed to determine the stationary solutions and their almost-sure stability properties for nonlinear gyroscopic systems which are stochastically excited. In addition, mechanisms which give rise to stochastic bifurcations in nonlinear random dynamical systems will be studied.

- In the final part of this research, dynamic experiments will be conducted on a rotating shaft to verify the theoretical results obtained. These dynamics experiments will locate the stability boundaries and examine the nature of the nonlinear response. The numerical and experimental results will, in tum, guide the development and refinement of the theories to incorporate any new phenomena observed.

\section{University Of Illinois}

Dept of Mechanical and

$\$ 82,179$

Industrial Engineering

Urbana, IL 61801

\section{Origins of Asymmetric Stress-Strain Response in Phase Transformations H. Sehitog/u}

A number of uniaxial and stress state experiments on the NiTi alloys that are known to undergo thermo-elastic phase transformations were conducted. Unlike steels which exhibit virtually no recoverable transformation strains, the transformation strains in this class of materials are partially recovered upon unloading, depending on the applied strain levels. Using a servohydraulic intensifier, a servohydraulic test machine, and a novel pressurized test chamber; pressures of $750 \mathrm{MPa}$ and axial stresses of almost any magnitude are simultaneously generated and applied to the gage section of a solid, cylindrical NiTi specimens. The work utilizes a robust internal load cell that can measure axial forces without the effect of seal friction and demonstrate innovative ways of calibrating this load cell, and methods of axial and circumferential strain measurement in a pressure environment and verify accuracy of these results. Constitutive models proposed in the literature for thermo-elastic transformation were evaluated in light of these results. The current models predict that the volume fraction of martensite is solely dependent on the effective stress. Our experimental results indicate that there is a dependence of the transformations strain on the hydrostatic stress component with strong asymmetry in tension versus compression. In view of these experimental findings, new transformation models are being developed incorporating the low symmetry of the twinning planes. The stress-induced phase transformation of CuZnAl was also found to be stress state dependent but less so than NiTi. 


\section{Johns Hopkins University}

Mechanical Engineering Dept

$\$ 120,163$

Baltimore, MD 21218

\section{Two-Fluid Averaged Equations for Multi-Phase Flow}

\section{A. Prosperetti}

The ultimate purpose of this study is to develop accurate averaged-equations models of disperse multi-phase flows of engineering significance. For this purpose, a method has been devised by which direct numerical simulations are used systematically to derive -- rather than "guess" -- the terms that are left undetermined by the averaging procedure.

In this work a particular emphasis is placed on the calculation of terms involving spatial derivatives of the averaged quantities, that are essential for the stability and hyperbolicity of the model. In the past no technique capable of giving insight into these terms has been available, other than at very low volume fractions.

The main difficulty that had to be overcome in the development of this approach has been the ability to calculate such terms -- which inherently refer to non-uniform suspensions -- relying on techniques developed for uniform suspensions. This was an unavoidable step as it is only in the uniform case that sufficiently powerful computational algorithms are available. A new approach capable of achieving this end has been developed and has been successfully implemented for the case of heat conduction in a composite. Work on Stokes flow is near completion, and the opposite limit of high-Reynolds-number flow has also been addressed with promising results.

\section{Robert H. Kraichnan, Inc.}

369 Montezuma 108

$\$ 77,370$

Santa Fe, NM 87501-2626

\section{Turbulence Theory and Reduced Hydrodynamics}

\section{R. Kraichnan}

Turbulent flow is omnipresent in geophysics and energy-producing devices. Atmospheric turbulent transport plays an essential role in the movement of heat, moisture, and pollutants. Turbulent flows represent an enormous amount of data. Both for practicable computation and for physical understanding, it is necessary to extract the essential information in compact form. This project explores novel approaches to economical description and computation of turbulence. Explicit statistics have been deduced theoretically for the spottiness of small scales of a contaminant carried by turbulence. This is the first such success for a turbulence problem, and has sparked an intense and continuing international follow-up effort. The theoretical work has been used under this Grant to guide computer simulations of unprecedented resolution. Recent attention has been focussed on intermittent velocity fields. A result of this project should be improved, economical computation of contaminant dispersal and mixing. Current work has extended the theoretical understanding of intermittency) to the prediction of new features (including a new scaling range) in one-dimensional model flow equations.

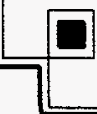




\section{Lawrence Berkeley National Laboratory}

Dept of Physics

University of California

Berkeley, CA 94720

\section{Studies in Nonlinear Dynamics}

\section{A. Kaufman, R. Littlejohn}

This research is devoted to the development of new methods for analyzing the evolution of waves and other dynamical systems. The primary approaches are Hamiltonian and Lagrangian, utilizing phase-space-geometric methods and variational principles. The wave-fields studied are typically multi-component, so that polarization and geometric-gauge concepts are important. Particular emphasis is placed on mode-conversion processes. Current projects include: (1) The resonant interaction and energy transfer between an eastward propagating equatorial-wave-guide Rossby-gravity wave and a westward propagating coastal Kelvin wave; (2) Auto-resonant phase-locking of such guided waves by nonlinear freqency shifts; (3) Tangential mode conversion, in which ray curvature dominates the interaction process; (4) Correlated emission of waves in a mode-conversion region; (5) Effect of polarization-dependent dissipation on the double-conversion process.

Another project involves the exploration of the theoretical underpinnings of non-imaging optics, a new field of optics in which the emphasis is typically on the detection, transport and/or concentration of radiant energy, rather than the formation of an image. Nonimaging optics is important in solar energy technology, infrared countermeasures, and other areas. Our work in recent years has concentrated on an elucidation of the fundamental principles of nonimaging optics, starting from electromagnetic theory. This is another area in which phase space representations of wave fields and other phase space techniques have been important. For example, in a recent paper we have shown how one may measure the phase space distribution of an optical field.

\section{Lawrence Livermore National Laboratory}

Laser Programs, L-482

$\$ 100,000$

University of California

Livermore, CA 94551

\section{Steady State and Transient Nucleation Kinetics G. Wilemski}

This research addresses several fundamental issues in homogeneous nucleation theory. Currently, the appealing simplicity and wide applicability of classical nucleation theory is offset by its inaccurate predictions of nucleation rates. On the other hand, new molecular theories of nucleation appear capable of providing greatly improved predictions, but practical application of these theories is currently restricted to the simple rare gas systems. The status of binary nucleation theory is further clouded by the inability to test theoretical predictions about the compositional structure of mixed nanodroplets because of the lack of direct experimental data on this phenomenon. The goals of this research are threefold: (1) to provide the theoretical underpinning for a powerful new analysis technique, small angle neutron scattering (SANS) from

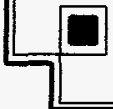


nanodroplet aerosols, that can probe the compositional structure of nanodroplets, (2) to explore ways of bridging the gap between fundamental molecular nucleation theories and phenomenological approaches based on thermodynamic reasoning, and (3) to test and improve binary nucleation theory.

The importance of small angle neutron scattering from aerosols (aerosol SANS) stems from its potential to probe directly the microscopic structure of the nanometer-sized droplets. Particles in this size range are the first condensed phase fragments to appear during aerosol formation. Aerosol SANS measurements also yield considerable detail about the particle size distribution of the aerosol allowing stringent testing of theories of nucleation and droplet growth under conditions of high supersaturation. The aerosol SANS technique relies on a supersonic nozzle expansion to reliably produce a steady flow of aerosol. The measured scattering patterns are anisotropic because the high directed velocity of the particles Doppler-shifts the momentum of the scattered neutrons. The basic theory needed to properly analyze these scattering patterns has been developed, and refinements are being pursued. Modeling of both unary and binary nucleation and droplet growth is also underway to both predict and interpret the experimental scattering patterns. Nucleation rates in nozzles are extremely high, and the corresponding critical clusters contain ten to twenty molecules. To improve the theory of nucleation under these extreme conditions an effort is being made to develop theoretical expressions for cluster evaporation rates based on computer studies of small clusters of more complex molecules such as water and methanol. Theoretical work is also being done to test and improve various aspects of binary nucleation theory. The birth-death equations are accurately solved numerically to simulate binary nucleation kinetics for fluid systems with strong compositional surface enrichment, partial miscibility, and significant vapor phase nonideality. These results are then used to assess the accuracy and applicability of various approximate analytical expressions for the rate of binary nucleation. The results of these investigations also provide valuable input for the aerosol SANS modeling work.

\section{The Lovelace Institutes}

Institute for Basic and Applied

Medical Research

Albuquerque, NM 87108

Two-Phase Flow Measurements by Nuclear Magnetic Resonance (NMR) E. Fukushima, S. Altobelli, A. Caprihan

The objective is to apply nuclear magnetic resonance (NMR) to study how mixtures of different phases diffuse and flow. NMR has been used to non-invasively measure concentration and velocity of suspensions undergoing steady flows at Lovelace. A future aim is to measure flow parameters for both phases of a solid-liquid mixture by utilizing the relaxation rate difference between the two components. Because the usual NMR measurements, especially imaging, are slow, an obvious goal is to develop methods to take data much faster. One approach to this objective is to develop a hardware compensation circuit to reduce the distortion of the magnetic field gradient pulses caused by eddy currents induced in conducting surfaces in and around the NMR sample. The other is to develop dedicated hardware that performs rapid computations resulting in real-time image reconstruction from the data. Vibrating beds of granular material are difficult to study by conventional optical methods because of their opacity. They are not easy by NMR, either, because the flow is not in steady state, i.e., the sample configuration changes with

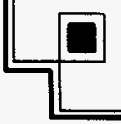

Engineering Research 
time even though the variation is periodic. The periodic nature has been used to gate the imaging sequence to the motion resulting in 3-D images of shaking granular beds. In this way, sharp spikes of particles on the surface of the bed have been imaged for the first time. An ultimate target of this project is to be able to measure a periodic nonsteady flow such as bubbly flows and turbulence.

\section{McDermott Technology, Inc.} Research and Development Division

Alliance, $\mathrm{OH} \quad \mathbf{4 4 6 0 1}$

\section{Investigation of Law Temperature Time Dependant Cracking W. A. Van Der Sluys}

This project is investigating the metallurgical and mechanical phenomena associated with the occurrence of time dependent cracking of carbon steels at temperatures between $620^{\circ} \mathrm{F}$ and $680^{\circ} \mathrm{F}$. Time dependent cracking failures of carbon steel piping in this temperature range have been experienced in the fossil power industry for the past twenty years. Moreover, the catastrophic failures occurred suddenly, creating a serious threat to human safety and substantial monetary losses. Research programs conducted to address this problem have demonstrated that this type of failure is proceeded by time dependent crack growth. Recent field failures demonstrate that our understanding of the fundamental metallurgical and mechanical parameters controlling this type of failure, are insufficient to eliminate it from the field.

This project is therefore focused on the metallurgical phenomena associated with low temperature time dependent crack growth in carbon steels. The project has been separated into two phases, the physical metallurgy phase associated with time dependent changes occurring in the crack tip region, and critical mechanical metallurgy experiments to quantify the time dependent cracking susceptibility of carbon steels.

Time dependent cracking is strongly linked to the content of residual elements in the steel composition. To address the composition issue, four heats of steel with varying combinations of residual element contents have been obtained. The compositions of these heats are such that correlations between cracking susceptibility and bulk residual element are being studied.

\section{University Of Maryland}

Dept of Mechanical Engineering College Park, MD 20742

\section{Characterization of Metal Cutting Dynamics B. Berger, I. Minis}

The development of energy efficient machine tools which produce parts of high quality and require minimum intervention requires the capacity to identify precursors of unstable, chatter states and integrate such identifiers into control system algorithms. Large amplitude oscillations associated with chatter adversely effect surface finish, dimensional accuracy and tool wear often resulting in tool breakage. 
This research effort has addressed the fundamental issues involved in the identification and control of chatter through: (i) creation of a data base of over 200 cutting experiments comprising $16 \cdot 10^{7}$ separate measurements for ranges of values of depth of cut, turning speed and feed rate; (ii) analysis of time series in the data base with a variety of techniques including false nearest neighbors, mutual information, singular value decomposition, wavelet and polyspectral methods; (iii) identification in the data analysis of three non-dimensional measures of the cutting state which are sufficiently robust for use in the on line control of the cutting machine; (iv) incorporation of the measures of the cutting state into the control system of a Harding Superslant $A B C N C$ lathe.

The three measures of the cutting state, (iii), are based on the ratio of singular values of an unsymmetric Toeplitz matrix of third order cumulants, a wavelet decomposition of cutting force data and a cutting force averaging process, respectively.

\section{University Of Maryland \\ Dept of Electrical Engineering \\ College Park, MD 20742 \\ Mathematical Models of Hysteresis \\ I. Mayergoyz}

$\$ 104,000$

This research is concerned with nonlinear diffusion of electromagnetic fields in hysteretic media. Examples of such media are ferromagnetic conducting media with magnetic hysteresis and superconducting media with sharp or gradual resistive transitions.

The main research objectives of the project can be briefly summarized as follows: development of analytical techniques for the calculation of eddy currents in magnetic media with hysteresis for linear polarizations of magnetic field (scalar problems), analysis of eddy currents in isotropic and anisotropic media with hysteresis for vector polarizations of magnetic field (vector problems), further development of mathematical models for hysteresis in superconductors with gradual and sharp resistive transitions, development of nonlinear impedance type boundary conditions for hysteretic media and their finite element implementation. It is hoped that, as a result of this research, new analysis techniques will be developed which will find numerous applications in such areas as nondestructive testing, magnetic recording, design of superconducting magnets, induction heating, electromagnetic shielding, evaluation of power losses, etc.

\section{University Of Maryland}

Electrical Engineering Department

$\$ 136,749$

\section{Baltimore, MD 21228}

\section{Pulse Propagation in Inhomogeneous Optical Waveguides \\ C. Menyuk}

Our current investigations are focused on two overlapping subjects. The first is dispersion-managed solitons. It has recently been discovered that the same sort of dispersion map that has trditionally been used for non-return to zero signal transmission can be productively

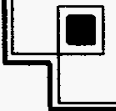


used for what we refer to as disperiosn-managed solitons. These are signal pulses that periodically return to their initial shape after every pas though the dispersion map, although they undergo a large amount of distoriton during each pass. We are investigating these signals both theoretically and experimentally; to date, we hold the world's record for distance $x$ time products in an unguided system, and we have developed new procedures for calcualting the noise contribution that we believe could significantly impact these calculations for all communication formats. The second subject concerns randomly varying birefringence in the optical fiber as it interacts with polarization-dependent loss, polarization-dependent gain, and polarization mode dispersion. Since the split step method cannot be used to effectively address these issues in an environment with wavelength division multiplexing, we have established a new approach that follows the Stokes parameters of each channel without addressing the intersymbol interference in each channel.

\section{University Of Maryland}

Dept of Mechanical Engineering

College Park, MD 20742

\section{Contaminant Dispersal in Bounded Turbulent Shear Flows J. Wallace, P. Bernard, L. Ong}

The purpose of this project is to better understand the physics of scalar transport and to develop a methodology for the accurate prediction of scalar fields in highly sheared environments, including the development of a new clasds of models that more accurately mimic the dynamical processes affecting plume development. Of particular interest is the wall-normal diffusion of scalars and its connection to vortical structures in the wall region, and the efficacy of the transport models in both canonical boundary layer flows as well as in complex flow geometries with significant flow separation. Towards these objectives, closely coordinated direct numerical simulations (DNS) and windtunnel experiments of evolving line- source plumes have been conducted.

Simultaneous flow visualization (using a laser light- sheet tomographic system) and velocity measurements (using a 4-sensor hot-wire probe) were performed in a turbulent boundary layer. A smoke plume seeped into the flow from a wall slot in the windtunnel idealized the dispersion of a passive scalar pollutant released from a line source in the atmospheric surface layer. These experiments have yielded detailed concentration contour maps as well as profiles of the concentration fluxes that have been used for model development and verification. Quasi-three-dimensional flow visualizations have been conducted with light sheets oriented in the spanwise direction. Reconstruction of three-dimensional surfaces of constant concentration has been accomplished. Finally, measurements of the concentration and concentration flux fields in a boundary layer with a two-dimension square obstacle on the wall have been carried out. This flow is an idealization of an urban canopy with a line scalar source at the wall.

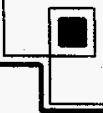


Massachusetts Institute Of Technology

Department of Chemical Engineering

$\$ 271,971$

Cambridge, MA 02139

\section{Synthesis and Optimization of Integrated Chemical Processes}

P. Barton, L. Evans

The goal of this research program is to develop novel systematic methods for the design and operation of chemical processes. As the chemical industry is one of the largest consumers of energy in the US, it is important to find efficient and creative computer-aided strategies for improving the efficiency of chemical manufacturing systems. One research thrust is addressing the need for process design and optimization technologies for batch/semi-continuous processes, which are increasingly important as the US shifts to the production of small quantities of high value added products. Research is focusing on design tools for the rapid development of efficient processes. The approach employed is based on the derivation of targeting models, which are simplified models that can rapidly generate a number of promising design alternatives for more detailed study. Since the targeting models also yield a rigorous lower bound on the cost of any potential design, this information can be used to justify and guide subsequent optimization of the design. In addition, the targeting models can be coupled with more detailed dynamic models to derive the first rigorous approach to address simultaneously the discrete and dynamic decisions in the optimization of a batch process design.

Secondly, complementary research is pioneering technologies for the optimization of large-scale dynamic systems, particularly focusing on discontinuous problems (e.g., hybrid discrete/continuous systems, inequality path constraints). Applications of interest include the design of optimal plant-wide operating policies for batch processes, or optimal operating procedures for start-up, shut-down and changeovers in continuous processes. A theoretical thrust focusing on discontinuous problem formulation, structure and classification, is accompanied by a numerical thrust that transforms the theoretical results into robust and efficient algorithms for large-scale problems.

\section{Massachusetts Institute Of Technology}

The Energy Laboratory

$\$ 364,736$

Cambridge, MA 02139

\section{Metal Transfer in Gas Metal Arc Welding in the High Current Regime \\ T. Eagar}

Two coordinated modeling efforts were started in 1997. The overall goal of the work is to explain droplet detachment and weld pool behavior in higher current modes typical in high productivity industrial applications.

The first effort is to develop a dynamic model for simulating electrode melting, drop formation, and drop detachment. Existing experimental results covering a wide range of welding parameters in different transfer modes were reviewed. Heat input, contact tube to workpiece distance, and shielding gas composition are parameters that will be included in ongoing modeling work. Emphasis will be given to the physical effects which cause the transition from globular to spray

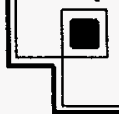

Engineering Research 
transfer. The model should predict size, frequency and heat content of the transferred drops over a wide range of parameters.

The second model will predict the effect of molten metal flow, surface effects, and droplet impingement on the weld pool. The model will contribute to control of weld defect formation such as humping, tunnel porosity and undercutting. A simplified fully-coupled model for the weld pool was developed, and it suggests that the weld pool tends to behave as a thin liquid film at high currents. This could explain the "finger penetration" phenomenon.

\section{Massachusetts Institute Of Technology}

Francis Bitter Magnet Laboratory

$\$ 93,324$

Cambridge, MA 02139

Cryotribology (Low-Temperature Friction and Wear): Development of Cryotribological Theories and Application to Cryogenic Devices

Y. Wasa, E. Rabinowicz

The main objective of this project is to develop a better understanding of mass transfer between bubbles and impure liquids. New techniques will be developed that can be used to analyze mass transfer in a broad range of problems. The focus is on bubbles between $0.5 \mathrm{~mm}$ and $2.0 \mathrm{~mm}$ in water. Issues of particular interest are the transient period during which surfactants adsorb onto the bubble and the effect of the transient on bubble dissolution.

The scientific approach involves laboratory experiments and numerical simulations. Experiments will be done to measure the velocity and dissolution of bubbles in both pure water and water containing controlled amounts of surfactants. The numerical simulations will provide information about the flow and concentration fields around the bubble and the dependence of the transient period on surfactant sorption kinetics. A new numerical technique will be used to simulate mass transfer between a bubble and the surrounding liquid.

Computer codes for the solution of the Navier-Stokes and mass transfer equations have been developed and mass transfer results have been obtained for Peclet numbers of $O(102)$. A Monte Carlo code for larger Peclet numbers is being developed. The equipment for the experiments has been constructed.

\section{Massachusetts Institute Of Technology}

The Energy Laboratory

$\$ 198,451$

Cambridge, MA 02139

\section{Modeling and Analysis of Surface Cracks}

D. Parks, F. McClintock

We are developing a mechanics basis for analyzing the fracture behavior of cracks located on or near the fusion zones of structural weldments. Such welds are often characterized by<smiles>C=Cc1ccccc1C=C</smiles> 
significant strength mismatch between base plate and weld metal, as well as by local strength gradients associated with metallurgical details of the heat-affected zones. Moreover, the local gradients in microstructure, and the accompanying gradients in material resistance to both ductile hole growth and cleavage fracture mechanisms provide additional complexity, compared to the corresponding fracture mechanics models of macroscopically homogeneous crack-tip microstructures and properties.

Under macroscopic mode I loading, strength-mismatched interface crack-tip stress and deformation fields show considerable differences from the corresponding fields in mechanically homogeneous media. In particular, both triaxial stress and plastic strain levels in the softer domain (e.g., an undermatched baseplate) are elevated. Families of mismatched fields have been characterized by finite element and slip-line solutions, and have been shown to apply from small-scale yielding through fully-plastic conditions.

The mismatched fields are being coupled with local models of cleavage and ductile fracture in the inhomogeneous crack-tip region, and the results compared with experiments on both model weldments created by diffusion-bonding and with actual welds in A710 steel.

\section{Massachusetts Institute Of Technology}

Dept of Chemical Engineering

$\$ 383,422$

Cambridge, MA 02139

\section{Development of Principles \& Methodologies of Metabolic Engineering G. Stephanopoulos}

Metabolic Engineering is an emerging field of biotechnology aiming at the directed modification of the metabolic pathways of microorganisms, plants and animals using recombinant DNA technology. The overall objective is to achieve overproduction of fuels, chemicals and materials, or biosynthesis of novel products through the amplification of selected biochemical reactions or the introduction of new biosynthetic pathways in metabolic networks. The experimental techniques of metabolic engineering are derived from applied molecular biology and are well advanced. However, interactions of metabolic pathways and the general principles governing metabolic fluxes in-vivo are poorly understood. The goal of this research is to contribute to the development of the tools and principles that elucidate the control of flux in metabolic networks. As the problem of determining flux control distributions at the individual reaction level is too complex and experimentally intractable, our approach has examined the control of flux exercised by groups of reactions. Concepts from metabolic control analysis have been extended to groups of reactions in order to identify the group exercising the strongest degree of control on the production flux. The approach is then repeated within the group until single reactions are identified that are of particular importance to the flux of product formation. This approach has been applied with success to the production of aromatic and aspartate aminoacids in Corynebacterium and yeasts.

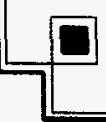




\section{Massachusetts Institute Of Technology ${ }^{1}$}

Dept of Chemical Engineering

Cambridge, MA 02139

\section{Los Alamos National Lab ${ }^{2}$}

MEE-9

Los Alamos, NM 87545

\section{Sandia National Laboratories ${ }^{3}$ \\ Engineering Sciences Center \\ Albuquerque, NM 87008-0834}

\section{Macrostatistical Hydrodynamics}

\section{H. Brenner', A. Graham², L. Mondy ${ }^{3}$}

Experimental, analytical, and numerical studies of falling-ball 'tracer' particle dynamics in concentrated suspensions composed of dispersed, neutrally-buoyant spheres and rods comparable in size to the tracer have been continued and extended. Falling ball viscometry was shown to be a useful technique to measure the apparent viscosity of suspensions of particles without unduly disturbing the initial configuration of the suspended particles. The pressure 'drop' across a ball falling through a quiescent suspension, which constitutes another useful dynamical suspension parameter, was measured and, in moderately concentrated suspensions, found to agree well with theoretical results for the comparable pressure drop in a homogeneous Newtonian liquid. For a ball falling in a highly concentrated suspension, the pressure drop seems to be a sensitive measure of phase slip at the containing cylinder walls. Also, spinning-ball rheometry in quiescent suspensions has been developed as a useful adjunct to the falling-ball rheometric studies. This technique provides a sensitive measure of phase slip at the spinning ball's surface, as well as another experimental benchmark for ongoing theoretical investigations of slip in disperse systems. Finally, in collaboration with The Lovelace Institutes, nuclear magnetic resonance imaging is being used to study flow-induced particle segregation ("hydrodynamic diffusion") in pipe flow. These data in inhomogeneous flows and complementary video imaging of individual tracer particles in homogeneous flows will provide much needed information on the effects of flow on particle interactions and effective rheological properties at the macroscale. For example, the dispersion in velocity of tracer particles in an otherwise transparent concentrated suspension undergoing homogeneous shear flow is currently being measured using techniques developed in earlier falling ball studies. The resulting information on the self-diffusion of a suspended sphere among like spheres is central to kinetic-theory-type models of suspension flow.

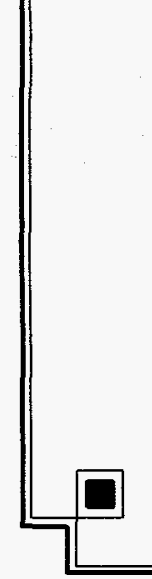


University Of Minnesota

Dept of Mechanical Engineering

Minneapolis, MN 55455

$\$ 137,999$

\section{Heat/Mass Transfer Enhancement in Separated and Vortex Flows \\ R. Goldstein}

The flow and heat/mass transfer around single short-step and uniform diameter circular cylinders are being studied. Flow visualization indicates that a short step diameter circular cylinder has a much more complex vortex system than a uniform diameter circular cylinder due to vortices formed on the steps of the cylinders. Velocity and turbulence measurements reveal that the interaction between the vortices formed on the steps of the cylinder and those formed near the endwall junction change the vortex shedding frequencies (Strouhal Number) in the wake flow. The measured local heat/mass transfer compares well with flow visualization and velocity measurements, and confirms the vortex flow patterns.

Energy (total temperature) separation associated with unsteady pressure fluctuations, induced by the convective movement of vortices has proved to have strong effects on heat transfer. Energy and total pressure separation in and around a free circular jet, including the effects of acoustic excitation, and the energy (total temperature) separation around a circular cylinder in high speed flow are being investigated. For the flow in the vortex ring structure around a jet, energy separation and the total pressure separation amplitude are greatly increased by acoustic excitation. This excitation is expected to have a strong effect on the jet impingement heat/mass which will be investigated. For the circular cylinders, the preliminary results show that the dominant transient recovery temperature frequency on the cylinder surface in the wake region matches the vortex shedding frequency.

\section{University Of Minnesota}

Dept of Mechanical Engineering

Minneapolis, MN 55455

\section{Thermal Plasma Chemical Vapor Deposition of Advanced Materials}

\section{J. Heberlein}

The objective of this project is to broaden the technical base for processing of diamond, yttria stabilized zirconia and nanophase composite materials.

The studies related to diamond deposition have concentrated on characterizing the liquid injection diamond deposition reactor using a combination of modeling and analysis of deposition results. The model has been completed as a fully three-dimensional fluid dynamics and chemical kinetics description of the reactor, including the atomization of the liquid precursor and the trajectories and evaporation of the droplets. Various operating conditions have been simulated, and it has been found that liquid precursors result in higher deposition rates if the liquid droplets reach the substrate boundary layer before evaporating. 
Investigations on yttria stabilized zirconia (YSZ) layers have concentrated on developing a deposition technique using our unique triple torch reactor for generating very dense films which can be used as electrolyte in solid oxide fuel cells. A fundamental limitation of plasma CVD has been found, and a new process, center injection low pressure plasma spraying, has been developed. Films have been deposited with this process at high rates $(>60 \mathrm{~m} / \mathrm{min})$ with porosities $<0.5 \%$.

The spatially and temporally resolved characterization of turbulent non-equilibrium plasma jets using laser scattering techniques developed at the Idaho National Engineering Laboratory has confirmed the discrepancies found at INEL. Research efforts are now concentrating on comparing assumptions required for the theoretical line shape derivation with the conditions present in the experiment.

\section{University Of Minnesota}

Dept of Aero Eng \& Mechanics

$\$ 120,000$

Minneapolis, MN 55455

\section{Lubricated Transport of Viscous Materials \\ D. Joseph}

The project has as its broad aim the understanding and technological development of lubricated pipelines of heavy oils in core annular flow, and the spontaneous lubrication of oil/water and water/oil emulsions and for slurry transport. The scientific approach relies heavily on experiments and direct numerical simulations. Analytically the problem involves the study of two-phase flows and deals with migration and segregation of lubricating fluid at the wall. The role of inertia in centering density matched core-annular flows and in levitating these flows off the wall when the fluids have different densities has been clarified in recent works sponsored under this grant. Experiments on the effects of different linings and surfactants on the fouling of pipe walls with hydrocarbons were carried out. A patent for a "method of preventing fouling of pipe walls for lubricated transport" was obtained for cement linings (U.S. Patent No. 5,385,175). Another patent for a non-fouling pressure tap is under consideration. It has been found that oil-in-water emulsions used as a coal substitute fluid and stable water-in-oil emulsions used in the processing of synthetic crude oils will lubricate when flow speed is above a critical one and other attainable conditions are satisfied.

\section{National Academy of Sciences- National Academy of Eng Washington, DC 20418}

\section{Third Annual Symposium on Frontiers of Engineering}

\section{J. R. Hunziker}

With advances in engineering moving increasingly across many facets of the profession; bringing together outstanding younger engineers from disparate fields and challenging them to think about developments at the frontiers of areas other than their own, could have a variety of desirable

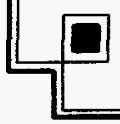


results. Such results include collaborative work, the transfer of new techniques and approaches across fields, and the building of contacts among the next generation of engineering leaders. This is the rationale for the Frontiers of Engineering Symposium Series.

The Third Annual Frontiers of Engineering Symposium was held September 18-20, 1997. The meeting brought together 101 engineers (ages 30-45), 45 from academia, 45 from industry, and 11 from government labs. With primary objectives as stated above, the scope of the meeting covered five topics: Biomechanics, Sensors and Control for Manufacturing Processes, Safety and Security Issues, Decision-Making Tools for Design and Manufacturing, and Intelligent Transportation Systems. The format of the meeting was to have formal presentations, typically by two to four individuals within each subject area, followed by a substantial question-and-answer and discussion period. Occasionally, panel discussions were employed to raise cross-cutting issues. The dinner speech by Philip Condit, Chairman and CEO of The Boeing Company, provided an opportunity for insights from and interaction with a highly-respected senior engineer.

The primary outcome of the meeting was the interaction it facilitated among the best young American engineers from different fields and sectors. Attendees mentioned how rare it is to have an opportunity, if they are academics, to talk to engineers from industry, and vice versa. They also noted that it was enriching to learn about advances in fields other than their own, and that they found the information useful to their own work. The meeting created the opportunity for sharing of research insights and techniques that can spur cross-field collaboration, stimulating leaps in engineering knowledge and applications that otherwise would take longer to achieve, if achieved at all.

The publication that resulted from this meeting is Frontiers of Engineering: Reports on Leading Edge Engineering from the 1997 NAE Symposium on Frontiers of Engineering. 1998. Washington, D.C.: National Academy Press. The publication was disseminated to the engineering and public policy community through hard copy and by posting on the Internet.

\section{National Academy of Sciences- National Academy of Eng National Research Council Washington, DC 20418

\section{Department of Energy Integrated Manufacturing Fellowship Program T. Rozzell}

Thirty-six three-year predoctoral fellowships in integrated manufacturing are in place administered by the National Research Council, under the aegis of the National Academy of Engineering, following national competitions. The objectives of the program are to create a pool of PhD's trained in the integrated approach to manufacturing, to promote academic interest in the field, and to attract talented professionals to this challenging area of engineering.

The fellowship program was conceived as one response to the loss of competitiveness of the United States in manufacturing. Two related aspects of the problem are the traditional separation of the product design function from the manufacturing function and the lack of an appreciation for the process of manufacturing as an integrated system.

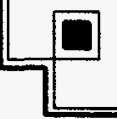


It is expected that the improved manufacturing methods which this fellowship aims to bring about will contribute to improved energy efficiency, to better utilization of scarce resources, and to less degradation of the environment.

\section{National Aeronautics and Space Adminstration} 600 Independence Avenue, S.W.

Washington, DC 20546

\section{Center for Aerospace Research \& Education for Minority Students at Southern Univ. I. Blankson}

Pursuant to an agreement between the Secretary of Energy and the Administrator of NASA, the recommended funds will support the education of minority students at Southern University (an $\mathrm{HBCU}$ ) in disciplines related to aeronautics and space sciences. Over the five years of this agreement, NASA will provide $\$ 2,500,000$. The education program will be coordinated with a research program addressing solid mechanics and finite element modeling, composite materials, aerodynamics and impact, as well as thermal sciences (heat transfer and fluid dynamics). In more detail examples of specific projects are improvement of satellite rendezvous maneuvers, optimization of gear design, analysis of the wear of diamond tools. Furthermore, these funds will be used to strengthen the undergraduate education in aeronautics at Southern University.

\section{National Center for Manufacturing Sciences}

Technology Sourcing

Ann Arbor, MI 48108

\section{Industrial Liaison Pilot Program \\ $J$. Sheridan}

The National Center for Manufacturing Sciences has been funded by DOE, Basic Energy Sciences, to direct a progream which will place employees of the National Laboratories at Industrial sites to work with their corporate colleagues on issues of importance to manufacturing industry. The goals of the program are to evolve expertise in a broad range of manufacturing issues at the National laboratories, identify fundamental DOE research needs, and build a longrange sustained commitment to collaboration on industrial issues both at DOE and within industry. Results from the Liaison Program will strengthen industry's energy efficiency in its operations by enhancing the qulaity of the total product and developing efficient production and management techniques. This will directly benefit American manufacturing competitiveness and will help DOE Basic Energy Sciences identify fundamental research needs in energy efficient industrial methodologies and technologies.

The pilot program is important. There are a number of potential risks and blockers which need to be assessed carefully before a large commitment is made. The Industrial Liaison Program (ILP) will place DOE laboratory workers into the industrial environment to focus the attention of the laboratories on industry issues. The worker will remain a full time employee of the Laboratory but the costs of the off-site per diem will be shared by the host company through a formula-based reimbursement.

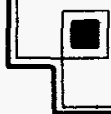




\section{National Institute Of Standards \& Technology}

Electromagnetic Technology Div

Boulder, CO 80303

Low-Frequency Noise of High Temperature Superconductor/Noble-Metal Interfaces and

High-Tc Superconducting Thin Films

J. Ekin, Y. Xu

The objective of this work is to characterize the low-frequency noise behavior of high-temperature superconductors/noble-metals interfaces, in order to provide superconducting electronics engineers with meaningful numbers to estimate the noise magnitude for a given interface design, and to mitigate the noise problems by better design and device fabrication process.

We will first measure the magnitude of noise as a function of the contact area. We will also modify the characteristics of interface conduction and study its effects on low-frequency noise. The interface can be modified by annealing the system in oxygen, or by engineering the interface in different crystal orientation, for example, along the c-axis direction or the ab-direction of the YBCO thin films. We plan to use both approaches.

Since the commencement of the program in March, 1997, we have been working on assembling the measurement system, a key element of which is a spectrum analyzer. We have also worked intensively on oxygen annealing of $\mathrm{YBCO} / \mathrm{Au}$ interface. We found that oxygen annealing substantially reduced the contact resistivity, and changed interface transport characteristics. Currently we are working on spectrum analyzer's GPIB interface.

\section{National Institute Of Standards \& Technology}

Thermophysics Division

$\$ 123,000$

Boulder, CO 80303

\section{Structure and Dynamics During the Formation of Dense Silica Gels}

H.J.M. Hanley

The project examines the gel structure of silica by SANS (small angle neutron scattering) and light scattering measurements. The goal is to extend the current understanding of how gels form on a microscopic scale, and to determine what specific changes are induced in the microscopic structure by the influence of an applied shear. Scattered intensity data were taken on the $30 \mathrm{~m}$ SANS instruments at the NIST Cold Neutron Research Facility. Samples were aqueous gels of Ludox silica particles of nominal diameter $24 \mathrm{~nm}$. The series of experiments were repeated with the silica samples loaded into the NIST Couette shearing cell. The results showed that shear most conclusively influences the structure of the gel. On the assumption that an investigation of decomposition of a system to a solid would lead to an insight into the gelation mechanism, a simulation of a two dimensional Lennard-Jones system was initiated. A striking similarity between the computer results and the SANS data was observed. A scaling relation for the time evolution of the structure factor of a decomposing system was proposed. SANS experiments carried out of $7 \mathrm{~nm}$ silica gels have verified that the scaling relation applies to the gelling system. Light scattering experiments on multiply scattering from the silica systems are in progress.

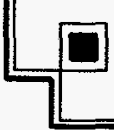




\section{National Institute Of Standards \& Technology}

Thermophysics Division

Gaithersburg, MD 20899

Boulder, CO 80303

Development of Measurement Capabilities for the Thermophysical Properties of Energy-Related Fluids

R. Kayser, W. Haynes

The major objectives of this new three-year project are to develop state-of-the-art experimental apparatus for measuring the thermophysical properties of a wide range of fluids and fluid mixtures important to the energy, chemical, and energy-related industries. The specific measurement capabilities to be developed are the following: Small-Volume, Dual-Cell DewBubble Point Apparatus; Heat-of-Vaporization Calorimeter and Effusion Cell for Vapor-Pressure Determinations; Solubility Measurements Using Magnetic Levitation; Thermal Diffusivity from Light Scattering; and Phase-Equilibria Apparatus for Azeotropic Aqueous-Organic-Salt Mixtures. These new apparatus will extend significantly the state of the art for properties measurements and make it possible to study a wide range of complex fluid systems (e.g., highly involatile, very insoluble, highly polar, electrically conducting, reacting) under conditions which have been previously inaccessible.

\section{The City University Of New York}

The City College

The Benjamin Levich Institute

for Physico-Chemical Hydrodynamics

New York, NY 10031

\section{The Rheology of Concentrated Suspensions}

\section{A. Acrivos}

This research program aims to investigate the flow of concentrated suspensions of non-colloidal particles from the fundamental point of view. Earlier studies by the principal investigator and his associates have shown that the rheology of such systems is strongly affected by the shear-induced migration of particles form regions of high shear to low and from regions of high particle concentrations to low which, by distorting the particle concentration profile, can lead to an erroneous interpretation of the experimental measurements pertaining to the effective viscosity of such systems. This shear-induced particle diffusion is also responsible for the phenomenon of viscous resuspension whereby, in the presence of shear flow, a settled bed of heavy particles can resuspend even under conditions of vanishingly small Reynolds numbers.

A theoretical model of shear-induced particle diffusion, which was developed earlier by the principal investigator was tested experimentally in various unidirectional flows by measuring the extent of viscous resuspension and comparing it to the theoretical predictions. Very good agreement was found between theory and experiments even in the case of flow in a tube where strong secondary flows are generated owing to the presence of a non-axisymmetric particle concentration profile. Also, a technique was developed, using LDA, for measuring the particle 
velocities in concentrated suspensions. This technique has been extended to measure instantaneous $3-d$ particle velocities and velocity fluctuations in concentrated suspensions undergoing a variety of shear flows in order to further test the predictions of the theoretical model. In addition, these studies are currently being extended to bi-dispersed suspensions involving particles of different sizes and densities.

\section{The City University Of New York}

The City College

Dept of Chemical Engineering

New York, NY 10031

\section{Partial Control of Complex Processing Systems}

\section{R. Shinnar, I. Rinard}

There are two research objectives. One is to understand the control of Fluidized Catalytic Crackers (FCC's), an important goal in itself. The second is to use the FCC as an example to learn about the control of complex chemical plants in which the number of variables to be maintained within acceptable limits exceeds the number of manipulatable variables available for control. This is known as partial control.

Work finished and published this year focuses on the impact of the process design on partial control. This was explored via the important concept of effective degrees of freedom. It was shown for the FCC that in the simplest case of operation in partial combustion, only one degree of freedom, the air feed to the regenerator, was required to control against changes in coking rate. The improvements in control obtained from adding other degrees of freedom such as catalyst recirculation rate, oil feed temperature, and heat removal using a catalyst cooler were studied to illustrate the tradeoffs between design and control. Finally, a general approach to the design of partial control systems was formulated.

Current work is underway in two areas. One is the exploration of the effects of model uncertainty on the control system design, again using the FCC as an example. Second is the formalization of the general approach and its application to control of systems and processes other than the FCC.

\section{The City University Of New York}

The City College

The Benjamin Levich Institute

New York, NY 10031

\section{Studies in Combustion and Explosion}

\section{G. Sivashinsky}

The objective of this research program is to investigate the influence of various aerodynamical, diffusive-thermal, radiative and reaction-rate factors on certain fundamental phenomena associated with the combustion and explosion of gaseous premixtures. Different modeling

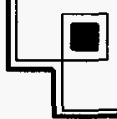


techniques will be employed to reduce the study of pertinent combustion systems to simple approximate problems tractable either analytically or numerically.

The first topic of the project concerns the problem of flame interaction with one- and multiplescale systems of eddies, and is motivated by the experimentally known phenomenon of flame extinction by turbulence. The results obtained show that the adiabatic high Lewis number premixed gas flame propagating through periodic vortical flow, constitutes a bistable system with a hysteresic transition between possible combustion modes. A mildly non-adiabatic flame may be quenced provided the flow-field intensity exceeds a certain critical level.

The second topic concerns the influence of natural conviction on the limits of thermal explosion. The problem is of direct relevance to combustion fundamentals associated with mine and grain silo explosions and knocks in internal combustoin engines. The explosion threshold is found to be strongly influenced by the flow field length scale and may be raised by the small-scale stirring and lowered by the large-scale one, compared to the case of stagnant premixture.

The third topic concerns the classical problem of the deflagration-to-detonation transition in pipes and dusted gases. The preliminary analysis based on a reduced reaction-diffusion-acoustics model showed that the transition is largely controlled by the friction-induced momentum losses, ignored in the previous attempts to describe the phenomenon. At sufficiently strong friction the normal Chapman-Jouget type detonation is found to undergo a jumpwise hysteresic transition to a low speed quasi-detonation dominated by dissipative effects.

\section{State University Of New York}

Department of Physics

Stony Brook, NY 11794

\section{Sub-Electron Transfer of Electric Charge in Semiconductor Nanostructures K.K. Likharev}

The goal of the project is to find the crossover between the continuous and discrete transfer of electric charge transfer in semiconductor nanostructures. Understanding of this crossover is of the key importance for the future development of nanoscale electronic devices. As a result of the theoretical effort during the first two years of the project, the charge discreteness has been calculated for three important models of nanostructures. The analysis has shown that the charge continuity cannot be provided by the electrostatic screening alone; another key condition is the smallness of the electron energy relaxation distance in comparison with the sample length. Experimental techniques to measure the charge discreteness via the intensity of non-equilibrium fluctuations in nanostructures have been developed. Initial measurements of the fluctuations have been carried out for p-doped channels of SiGe MESFETs and MOSFETs, with gates typically 30 um wide and 5 um long, at temperatures from 4 to $300 \mathrm{~K}$. To circumvent the 1/f noise which was dominant in first experiments, the experimental set-up is being extended to the $1-\mathrm{GHz}$ frequency range.

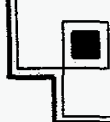


Thin-Film Characterization and Flaw Detection

\section{J. Achenbach}

This work is concerned with the determination of the elastic constants of thin films deposited on substrates, with the measurement of residual stresses in such films and with the detection and characterization of defects in thin film substrate configurations.

There are many present and potential applications of configurations consisting of a thin film deposited on a substrate. Thin films that are deposited to improve the hardness and/or the thermal properties of surfaces are of principal interest in this work. Thin film technology does, however, also include high Tc superconductor films, films for magnetic recording, superlattices and films for band-gap engineering and quantum devices. The studies carried out on this project also have relevance to those applications.

Both the film and the substrate are generally anisotropic. A line-focus acoustic microscope, is being used to measure the speed of wave modes in the thin film/substrate system. This microscope has unique advantages for measurements in anisotropic media. Analytical and numerical techniques are employed to extract the desired information on the thin film from the measured data. Recent results include: (1) use of multiple wave modes to determine thin film constants, (2) measurements of superlattice film constants, and (3) investigation of the effect of surface roughness.

\section{Northwestern University}

Dept of Chemical Engineering

Evanston, IL 60208

\section{Theory of Subcooled Boiling}

\section{S. Bankoff, S. Davis}

Subcooled boiling is perhaps the most efficient means of steady high-heat-flux transfer from solid surfaces, such as in rocket motors and heat treatment of metals. The growth and collapse of small bubbles while attached to the heating surface will be studied analytically and numerically. When the critical heat flux is exceeded, subcooled film boiling results. The stability of the resulting vapor film, involving momentary contacts or evaporating liquid tongues with the hot solid wall, and the criteria for growth of these contacts determine the minimum film boiling temperature. To begin with, two problems have been considered. The first paper deals with a circular, evaporating liquid wedge, which oscillates in radius, on a hot horizontal plate. This simulates the periodic dryout and rewetting by a thin liquid film under a growing-and-collapsing bubble in subcooled nucleate boiling. The second problem has led to a long-wave evolution equation for a thin vapor film on a horizontal heated plate, in subcooled film boiling. Linear

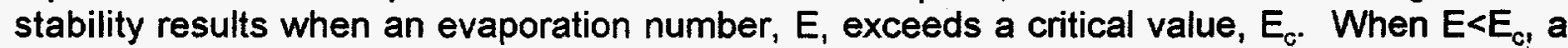
pitchfork bifurcation is found, and a new evolution branch is found. Work is continuing on both of these problems.

$\square$

Engineering Research 


\section{Northwestern University}

Dept of Eng Sci \& App Math

$\$ 64,114$

Evanston, IL 60208

\section{Complex Interfacial Flows \\ M. Miksis}

The research here in the first year of this grant will be directed into two areas. The first concerns the spreading of a liquid along the interface of a second liquid. Here we will be concerned with the stability and dynamics of the liquid interfaces. In particular, we will address the question of how the physical parameters in this problem influence the spreading. Second we will be concerned with the dynamics of foams. This study will build on our current work on modeling the microscopic behavior of foam flow in porous media and on the dynamics of thin films. Both of these problems are directly related to the clean up on both land and water of hazardous waste spills and enhanced oil recovery.

\section{Northwestern University}

Dept of Chemical Engineering

Evanston, IL 60208-3120

\section{Fragmentation and Dispersion of Powdered Solids in Viscous Liquids J. Ottino}

Mixing processes are ubiquitous in industry. Our aim is conduct broad-based basic work in the area of mixing with the goal of producing useful knowledge to wide array of users. Work may be divided into Mixing of Viscous Liquids and Powdered Solids and Mixing of Powders. Substantial progress has been made on all fronts. Computational studies have been completed in the area of aggregation in regular and chaotic flows [1]. Work on fragmentation - including erosion -- has been completed as well [2]. We have also made substantial progress in the development of a model dealing with liquid-liquid mixing processes involving drop breakup and coalescence [3]. Work carried under The Mixing of Solids heading, initially perceived as an extension of on our work in suspensions, is quickly becoming a large and distinct area. Substantial progress has been made in this area as well, particularly for the case of non-cohesive materials. We have shown that slow mixing processes can be modeled as a succession of avalanches and that processes can be divided into geometrical and dynamical parts. In depth aspects of dynamical aspects - leading for example to segregation effects -- can be incorporated by means of molecular dynamic simulations.

[1] S. Hansen and J.M. Ottino, Aggregation and Cluster Size Evolution in Non-Homogenous Flows, J. Colloid and Int. Sci., 179, 89-103 (1996).

[2] S. Hansen and J.M. Ottino, Agglomerate Erosion: A Non-Scaling Solution to the Fragmentation Equation, Phys. Rev. E, 53, 4209-4212, 1996; S. Hansen and J.M. Ottino, Fragmentation with Abrasion and Cleavage: Analytical Results, Powder Tech. (1997), to appear. 
3] J. M. Ottino, P. de Rousell, S. Hansen, and D.V. Khakhar, Mixing and Dispersion of Viscous Fluids and Powdered Solids, Advances in Chemical Engineering submitted (1997).

[4] D.V. Khakhar, J.J. McCarthy, T. Shinbrot, and J.M. Ottino, Transverse Flow and Mixing of Granular Materials in A Rotating Cylinder, Phys. Fluids, 9, 31-43 (1997).

\section{Northwestern University}

Dept of Eng Sci \& App Math

Evanston, IL 60208

\section{Stability and Dynamics of Spatio-Temporal Structures H. Riecke}

The objective of this project is to contribute to the understanding of the dynamics of spatial structures and of spatio-temporal chaos as they arise in physical systems ranging from flows in fluids and in granular media to lasers and coupled oscillators.

This task is approached by investigating paradigmatic cases that address fundamental and applied issues. Fundamental aspects to be investigated in particular are the characterization and classification of states that are disordered in space and time, and the possible mechanisms for the localization of structures in space. These studies are expected to be important also for applications. For instance, in wide-area lasers spatio-temporal chaos can reduce the quality of the laser beam and can even be detrimental to the device itself. To address such problems a detailed understanding of the origin and the nature of the chaotic state is necessary.

Using analytical and numerical techniques, a number of paradigmatic systems have been investigated. Convection driven by surface tension is important in materials processing; the stability of surface-tension-driven convection has been studied in detail. The effect of a simultaneous breaking of spatial and temporal symmetries has been investigated in a joint experimental/theoretical effort in fluid flow between rotating cylinders. Substantial progress has been made in the understanding of the stability of waves in anisotropic systems, e.g. in liquid-crystal convection.

Current studies of simple models investigate the possible mechanisms that are responsible for the localization of wave structures in periodically shaken granular media and the interaction between those structures ('oscillons'). The studies of spatio-temporal chaos focus now on a transition between ordered and disordered defect chaos in parametrically driven waves. 


\section{University Of Notre Dame}

Dept of Chemical Engineering

$\$ 79,873$

Notre Dame, IN 46556

Fundamental Study of Long-Short Interfacial Wave Interactions and Application to Flow Regime Development

M.J. McCready

Knowledge of flow regime is the most important issue in the design and operation of multiphase flow pipelines. Growth of interfacial waves that are long compared to the depths of the two phases is known to play an important role in regime transition from a stratified gas-liquid flow. Current procedures for predicting regime transitions use linear instability of one-dimensional momentum equations as a transition criterion. However, recent work has shown that the predictions of one-dimensional equations differ significantly from the full differential equations and further, that the development process of long wave disturbances is nonlinear.

In this project experiments and theory are being used to discover the flow parameters that control the growth rate of long wave disturbances. To allow for accurate comparison to theory, the system under study is a light hydrocarbon oil - water flow in a wide rectangular channel oriented close to horizontal. The long-wave - short-wave interactions that occur in these systems and can cause accelerated growth of long waves are being examined. Slight variations of the flow angle from horizontal allow a wide variation in the relative growth rates and speeds of long and short waves. Theory employs weakly-nonlinear simplifications of the complete Navier-Stokes equations and boundary conditions.

\section{Oak Ridge National Laboratory}

Engineering Physics

and Mathematics Division

Oak Ridge, TN 37831

Center for Engineering Systems Advanced Research (CESAR)

J. Barhen, H. K. Liu, L. Maya, N. Peterfreund, E. Oblow, L. Parker, V. Protopopescu, N. Rao, D. Reister

The primary mission of the Center for Engineering Systems Advanced Research (CESAR) is to develop, through innovative basic research, a core of excellence in intelligent systems technology. By combining long-range future-oriented R\&D efforts with near-term, sustained technology transfer to U.S. industry, and with undergraduate, graduate, and post-graduate student training, CESAR strives to enhance U.S. scientific and technological capabilities in strategic areas vital to energy independence, national security, and global competitiveness.

Currently, CESAR's efforts emphasize two basic research thrusts: computational nonlinear science and cooperating autonomous systems. The latter are characterized as systems that integrate perception, reasoning, and action to perform tasks under circumstances that are either insufficiently known in advance or dynamically change during task execution. Perception, reasoning, and action, as well as the integration of these modules into prototype working systems, present outstanding challenges for inter-disciplinary basic research. The CESAR

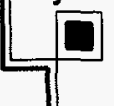

Engineering Research 
approach focuses the effort on high-priority research topics, such as multiple cooperating agents, multisensor fusion, and machine learning, and scopes the research activities so that integrated proof-of-principle experiments with the CESAR robot testbeds can be performed. Experimental paradigms include exploration and map-building using sonar and vision sensing, navigation in changing environments, and cooperative observation of multiple moving targets. Additional scenarios will include reliable 3-D world modeling based on laser range, sonar, vision, and novel hyperspectral sensors; and integration of high-level intelligent control with low-level reactivity behavior in robot architectures. In the area of computational nonlinear science, the main emphasis of the CESAR thrust is on developing innovative mathematical and computational tools to successfully address the selected "Grand Challenges". The proposed effort builds upon CESAR's unique advances in neural network learning, global optimization, solving inverse problems by optimal control techniques, and fusion rule estimation using empirical data. Promising new directions also being explored include quantum control of nanotechnology systems, as well as the emerging paradigms of optical and nanoelectronic-computing and information processing.

Technology transfer of CESAR innovations is rapidly growing, particularly to the automotive and petroleum exploration industries.

\section{Oregon State University}

Department of Mechanical Engineering

Corvallis, OR 97331

\section{Phase Change Phenomena in Enclosed Microstructures with Forced Flow}

\section{J.R. Welty}

This is a collaborative research effort involving Oregon state University and the Pacific Northwest National Laboratory in Richland, Washington. The objectives are the following:

1. To delineate how transport phenomena in microchannels differ from their macroscale counterparts. An understanding of, and ability to describe and predict behavior, is essential for the design of effective and efficient miniature thermal and chemical systems.

2. To investigate flow behavior within microchannels with widths of 100 microns and smaller under both isothermal and non-isothermal conditions with both single-phase (liquid) and two-phase (liquid-vapor) conditions present.

3. To understand and capture phase change phenomena along with dominant flow regimes in rectangular microchannels of various aspect ratios.

The experimental component of this effort involves using Particle Image Velocimetry (PIV) and Laser Induced Fluorescence (LIF) to obtain both a qualitative understanding of phase change phenomena and a quantitative understanding of the relationship between geometric characteristics, flow characteristics, imposed heat flux, and the attainable heat transfer and pressure drop. The simulation component involves the development and adaptation of Lattice-Boltzmann approaches to model both hydrodynamics and energy exchange in rectangular microchannels.

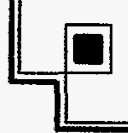




\section{Pennsylvania State University}

Mechanical Engineering Dept

University Park, PA 16802

\section{Research on Combustion-Driven HVOF Thermal Sprays G. Settles}

The High-Velocity Oxy-Fuel (HVOF) thermal spray process combines the fields of materials, combustion, and gas dynamics. It relies on combustion to melt and propel solid particles at high speeds onto a surface to be coated. The goal of this research is to understand and improve the HVOF deposition of corrosion-resistant coatings, which are important in many energy-related industries.

HVOF spraygun nozzle design and operating parameters have been found with which to vary the kinetic and thermal energies of the spray particles independently. For example, for sprayed coatings of 44 micron 316 Stainless Steel powder, the in-flight particle temperature is varied from $1600-2300 \mathrm{~K}$ and the particle speed is varied from $250-450 \mathrm{~m} / \mathrm{s}$. Through metallographic analysis, the resulting coating properties are seen to range from dense, high-quality coatings (low temperature, high speed) to oxide-bearing coatings of questionable utility (high temperature, low speed). The ability to tailor coatings to produce desirable properties, and knowledge of the wide range of coating properties possible from a given metallic powder, are important to the thermal spray field. Experiments and modeling using a broader range of sprayed materials are planned.

\section{Princeton University}

Dept of Mech \& Aero Eng $\$ 44,210$

Princeton, NJ 08544

\section{Unification of Stretched Flame Phenomena}

C. Law

The thermal structure of counterflow premixed and diffusion flames was experimentally and computationally determined to examine the response of flame structure to strain rate and pressure variations. For both near-equidiffusive and nonequidiffusive premixed flames, results show that variation of the thermal structures is much smaller than that of the strain rate, while the structural sensitivity also decreases with increasing pressure. For diffusion flames, results show that the flame structure at different pressures largely scale with the density-weighted strain rate. It is further demonstrated that stretch effects can be predicted with good accuracy by knowing the laminar flame speeds of the one-dimensional planar flame as a function of the system pressure and equivalence ratio.

The passive propagation of wrinkled, non-folding, premixed flames in quiescent and spatially periodic flow fields was investigated by employing the scalar field, G-equation, formulation. For the stretch-affected flame propagation mode, the resulting G-equation is in the general form of the Burgers' equation. Analytical solutions were obtained for several flame and flow types, describing the formation of cusps and their inner structure, and the augmentation of the average burning velocity through flame wrinkling. 


\section{Princeton University}

Dept of Civil Engineering

Princeton, NJ 08544

\section{Transport Properties of Disordered Porous Media From The Microstructure S. Torquato}

This research program is concerned with the quantitative relationship between transport properties of a disordered heterogeneous medium that arise in various energy-related problems (e.g., thermal or electrical conductivity, trapping rate, and the fluid permeability) and its microstructure. In particular, we shall focus our attention on studying the effect of: porosity, spatial distribution of the phase elements, interfacial surface statistics, anisotropy, and size distribution of the phase elements, on the effective properties of models of both unconsolidated media (e.g., soils and packed beds of discrete particles) and consolidated media (e.g., sandstones and sintered materials).

Both theoretical, computer-simulation, and experimental techniques have been employed to quantitatively characterize the microstructure and compute the transport properties of disordered media. Statistical-mechanical theory has been used to obtain n-point distribution functions and to study percolation phenomena in continuum random-media models. For example, the pore-size distribution, lineal path function, and the chord-length distribution function have been investigated and computed. This has led to accurate predictions of transport properties of realistic models of isotropic as well as anisotropic heterogeneous media. Cross property relations have been derived. Rigorous relations which link the fluid permeability to length scales obtainable from Nuclear Magnetic Resonance experiments and the effective electrical conductivity have been derived. Moreover, the effective conductivity has been related to the effective elastic moduli. Recently, 3-D images of a sandstone have been obtained using $x$-ray tomographic techniques and statistical correlation functions have been extracted from them. Recently, we have made progress on the inverse problem of reconstructing the structure of porous media from lower-order correlation functions.

\section{Purdue University}

School of Nuclear Engineering

West Lafayette, IN 47907

\section{Interfacial Area and Interfacial Transfer in Two-Phase Flow M. Ishii}

The objective of the research program is to develop instrumentation methods, experimental data base and models for describing the interfacial structure and behaviors of two-phase flows. In terms of the flow structure, the transverse distributions of the local void fraction, interfacial area concentration, fluid particle size and their axial development from the entrance to the exit will be the primary focal point of the experimental research. For the purpose of understanding the dynamic behaviors, the interfacial velocity, fluid particle coalescence and disintegration are studied. The axial changes in the distribution of void fraction and interfacial area give the information on the particle coalescence and disintegration. These are used to quantify the flow 
regime transitions. The multi-sensor probes are used together with hot-film probes for these measurements. The focus of the modeling effort is to develop an interfacial area transport equation which incorporates the mechanistic models for coalescence and disintegration of fluid particles. This transport equation describes dynamical change of the interfacial structure and replaces the conventional model based on flow regime transition criteria.

\section{Purdue University}

School of Mechanical Engineering

West Lafayette, IN 47907

\section{Critical Heat Flux in Micro-Channel Flow \\ I. Mudawar}

This project targets the development of a theoretical model for critical heat flux (CHF) from long heated walls in vertical flow. Flow boiling pattern is examined with the aid of photomicrographic and high-speed video imaging techniques in order to determine the CHF trigger mechanism. Close-up studies of the wall region have revealed features common to most bulk flow conditions. At fluxes below CHF, the vapor coalesces into a wavy layer which permits wetting only in wetting fronts, portions of the liquid-vapor interface which contract the wall as a result of the interfacial waviness. These waves are generated from the heater's upstream region with wavelengths following predictions based upon the Kelvin-Helmholtz instability criterion. Critical heat flux occurs when the pressure force exerted upon the interface due to interfacial curvature, which preserves interfacial contact with the wall prior to CHF, is overcome by the momentum of vapor at the site of the first wetting front, causing the interface to lift away from the wall. Recent studies have shown this interfacial lift-off criterion facilitates accurate theoretical modeling of $\mathrm{CHF}$ in both straight and curved channels. Present studies are focused on extending this model to highly subcooled conditions.

\section{Rensselaer Polytechnic Institute}

Dept of Mechanical Engineering,

Aeronautical Eng \& Mechanics

Troy, NY 12180-3590

Inelastic Constitutive Equation: Deformation Induced Anistropy and the Behavior at High Homologous Temperature

\section{E. Krempl}

Using experimental results obtained with computer-controlled, servohydraulic testing machines, continuum mechanics and materials science as backgrounds, constitutive equations (mathematical models of material deformation behavior that are used in stress and life-time analyses) are being developed with emphasis on two aspects: Deformation induced anisotropy for large deformation on the one hand and high homologous temperature on the other. Both areas extend the modeling capability of the previously developed "unified," state variable viscoplasticity theory based on overstress (VBO). 
A mathematical framework and a formulation for the representation of deformation induced anisotropy has been developed that includes the effects of texture. For large inelastic deformation an isotropic metal or alloy can be deformed into metal or alloy with elastic and inelastic anisotropy that depends on the deformation path. The compliance matrices are allowed to change according to an anisotropic Frederick-Armstrong growth law. Representations for initial isotropy and orthotropy have been found. Simulations of deformation induced anisotropy are underway.

The small strain version of VBO has been extended to high homologous temperature and applied to Alloy $600 \mathrm{H}$ at homologous temperatures above 0.7 . The model can simulate the experimentally observed creep and tensile behavior. It is also shown that the transition from the solid to the fluid state can be accomplished easily with VBO. It has also been applied to the modeling of solder alloys that operate high homologous temperature in the vicinity of ambient temperature. Creep and rate-dependent stress-strain behaviors were simulated well with VBO that had the minimum number of constants possible. This minimalist theory is also capable of modeling the Alloy $800 \mathrm{H}$ data that had previously been modeled with a version that had twice as many constants as the simplified version.

It was found that VBO could be modified easily to represent inverse rate sensitivity. This capability opens the door to the modeling of strain aging behavior that is found in most engineering alloys at some range of temperature.

\section{Rensselaer Polytechnic Institute}

Dept of Chemical Engineering

Troy, NY 12180-3590

Development and Use of Image Scanning Ellipsometer to Study the Dynamics of Heated Thin Liquid Films

\section{P. Wayner, Jr., J. Plawsky}

The physicochemical phenomena associated with fluid flow, change-of-phase heat transfer, and drying in ultra-thin (thickness less than $10^{-5} \mathrm{~m}$ ) aerogel and liquid films are being studied. In the thin liquid film systems, the interfacial, intermolecular, force field, which controls fluid flow and heat transfer, is a function of the film thickness profile. If the physical properties of the evaporating liquid film are known, the film thickness profile, measured experimentally, can be used to calculate the pressure field and the local evaporative heat transfer rate. To measure the film thickness profile, a novel ellipsometric technique (Image Scanning Ellipsometry, ISE) has been developed. ISE combines classical null ellipsometry with microscopic image processing, to determine simultaneously the thickness of the liquid film at all points on the substrate. Image processing helps identify the precise location on the surface of the liquid film which corresponds to the measured thickness. Thus a 2-D profile of the liquid film can be constructed. A second generation ISE employing enhanced resolution image acquisition and processing equipment has been constructed. The augmented processing power of the second generation instrument is expected to help plot more precise thickness profiles. Results obtained using the initial design were presented in Applied Optics, 33, pp 1223-1229, 1994, and, Physics Fluids, 6, pp 19631971 , 1994. Presently, the formation of thin aerogel films is being emphasized. The role of process parameters in the ambient drying proces, such as extent of silylation, aging time, $\mathrm{pH}$, and drying temperature, on the final film properties are being studied.

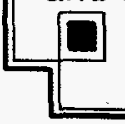


Rice University

Dept of Civil Engineering

Houston, TX 77251

A Novel Nonlinear System Identification Approach with Applicability to Aging of Energy Production and Distribution Systems

\section{P. Spanos}

Nonlinear forces acting on energy systems such as large offshore structures, are studied from a perspective of system identification. The nonlinearities may be induced by ocean waves, and they may become significant in several situations. They are not necessarily assumed to be in the form of Morison's equation. Various wave forces are examined. The force function is decomposed either into a set of base functions, or it is expanded in terms of the wave and structural kinematics. The resulting nonlinear system is decomposed in a number of parallel nonmemory, nonlinear systems, each followed by a finite memory subsystem. A Gram-Schmidt orthonormalization procedure is applied to decouple these subsystems; a frequency domain technique involving auto-spectra and cross-spectra is employed to identify the linear transfer function. In conjunction with this problem, an efficient algorithm for representing random processes by auto-regressive-moving-average digital algorithms is pursued for implementation into system identification problems. The analytical results are calibrated by Monte Carlo simulation studies.

\section{University Of Rochester}

Dept of Physics and Astronomy

Rochester, NY 14627

Flux Flow, Pinning, and Resistive Behavior in Superconducting Networks

\section{S. Teitel}

Vortex fluctuations play a dominant role in determining the phenomenological behavior of high temperature superconductors. In particular they are a major source of electrical resistance when an external magnetic field is applied.

Theoretical understanding of the effects of vortex fluctuations is therefore a key step in the technological application of the high temperature superconductors.

The system of many strongly interacting vortices, coupled to random material impurities, is a complex many-body statistical problem. To deal with this complexity, large scale numerical simulations are used to study behavior as temperature, applied magnetic field, material anisotropy, and random disorder are varied.

Recent efforts have focused on the behavior of three dimensional vortex lines in bulk superconductors, where a vortex line lattice forms at low temperature. Simulations indicate that for a finite temperature region above the vortex lattice melting temperature, the energy barrier for vortex line cutting is significant. The width of this region shrinks as the material anisotropyincreases. Such barriers to cutting can have a significant effect on vortex line diffusion, and hence flux flow resistance. New simulations are extending earlier equilibrium work to study non-equilibrium steady state dynamics, and to include the effects of random impurities.

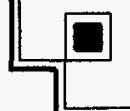

Engineering Research 


\section{University Of Rochester}

Dept of Physics and Astronomy

Rochester, NY 14627

$\$ 99,002$

\section{Direct and Inverse Problems in Statistical Wavefields \\ E. Wolf}

This research is chiefly concerned with determining the structure of fluctuating sources and also of scattering objects whose physical properties are random functions of position, from measurements of radiated and scattered fields. We have derived a new conservation law for fields generated by random, statistically stationary scalar sources of any state of coherence and we analyzed some of its implications on the phenomenon of correlation-induced spectral changes. We have extended the theory of diffraction tomography to make it possible to determine the degree of spatial correlation of a scattering potential of a random object from measurements of the crossspectral density of the scattered field in two planes, one on each side of the scatterer. We have also formulated a generalization of the optical crosssection theorem which in its usual form makes it possible to determine the scattering crosssection from the knowledge of the forward scattering amplitude. The generalization extends the theorem to situations where the incident field or the scatterer or both must be described statistically. We showed how the generalized theorem can be used to determine the structure of some random scatterers.

\section{The Rockefeller University}

Department of Physics

1230 York Avenue

New York, NY 10021

\section{Some Basic Research Problems Related to Energy}

\section{E. Cohen}

1. A very simple interpolation formula for the Newtonian viscosity as well as the visco-elastic behavior of monodisperse hard sphere colloidal suspensions has been derived theoretically for low as well as high concentrations. The formula has been adapted to micelles and is being tested for polymers. An extension to shear-rate dependent effects is in progress.

2. The investigation of Lorentz lattice gas cellular automata is continued. In this gas a point particle moves on the bonds of a lattice fully or partially occupied by deterministic scatterers of two types, which scatter the particle either to its left or to its right and change their scattering character after collision with the particle. Complex diffusion-propagation patterns of motion of the particle then emerge. This complex behavior of a very simple system could well be the prototype for a variety of phenomena found in nature. This is further investigated.

3. Dynamical weights using expanding Lyapunov exponents, rather than the usual probabilistic (Gibbsian) weights, can be employed to obtain the probability distribution of a many particle system in a nonequilibrium stationary state (e.g. a sheared fluid), which can be far from equilibrium. Successful applications of this new approach to obtain the properties of such a system have been made and are further pursued.

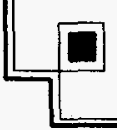




\section{Dynamically Active Scalars in Turbulent Combustion, Heat Transfer, and Geophysical Flows \\ A. Kerstein}

The principal focus of this research program is modeling of turbulent flows in which mixing modifies flow energetics, as in buoyant stratified turbulence and turbulent combustion. Turbulent motions are represented by mappings applied to a one-dimensional domain on which a velocity profile, and profiles of participating scalar quantities, are defined. The mappings are determined by turbulence energetics based on local shear and any dynamically active scalars. The mappings modify the velocity and scalar profiles, yielding a self-contained evolution process involving minimal empiricism.

Molecular transport (viscosity and species diffusion) are implemented concurrently with the mapping process in this fully resolved formulation. Models lacking full spatial resolution do not reflect the distinct roles of advection and molecular transport.

This formulation, denoted One-Dimensional Turbulence, has been applied to various constant-density and variable-density flows. The model reproduces key features of the turbulent cascade and the spatial structure (mean and fluctuation properties) of inhomogeneous shear-driven and buoyancy-driven flows.

Ongoing work focuses on flows of particular engineering and geophysical relevance. Salient topics include mixed-convection heat transfer, contaminant dispersion in the atmospheric boundary layer, and layering in double-diffusive convection.

\section{Science Applications Intl Corp}

Biotechnology Research and

Applications Group

McLean, VA 22102

Function-Based Biosensors for Use in Hazardous Waste Remediation

\section{J. J. Hickman}

\section{Research Objectives and Scientific Approach}

The goal of this work is to develop a new type of sensor capable of detecting toxins in hazardous waste effluents after remediation. This novel biosensor will monitor the changes in electrical signal between two neurons adhered to a uniquely patterned artificial surface in a defined growth media. The changes in electrical signal will correspond to the changes in neuron-neuron communication upon the introduction of a toxin. This type of sensor would be able to sense known as well as unknown toxins in hazardous waste effluents after remediation. 
Current Status of Project

Great progress has been made in several key areas via the commitment of SAIC internal research and development funds to jump-start and augment this program during the first year. Initial screening established extreme cases of self-assembled monolayer (SAM) modified surfaces which would either promote or impede neuronal growth on the substrates. These SAM's were then used to construct lithographically defined surface for neuronal patterning. A key area of progress was to establish a laser system that gave reproducible patterns. The collaborative effort with $\mathrm{NIH}$, NINDS has allowed the culture and electrophysiological characterization of rat hippocampal neurons on these high resolution patterned surfaces in defined media.

Preliminary cell culture results have indicated we have extended the longevity and fidelity on the patterns. We have applied new surface analysis tools in these investigations that have enabled a clearer understanding of the relationship between surface and cell response. In the second year of the project the primary goal will be extension of the in vitro culture system on the patterns to $>1$ month. Preliminary response of the rudimentary circuits to toxins will also be investigated. Finally, the initial solid-state system will be designed.

\section{Stanford University}

Dept of Mechanical Engineering $\$ 140,455$

Stanford, CA $94305-3030$

\section{Stress and Stability Analysis of Surface Morphology of Elastic and Piezoelectric Materials}

H. Gao, D. Barnett

The objective of this research has been to study morphological stabilities and instabilitites in elastic and piezoelectric solids. In morphologies are included surface shapes, cracks, and defect patterns. In the past year, we have developed techniques to study electrical nonlinearity near cracks in piezoelectric ceramics, and to study equilibrium and dynamic 2-dimensional arrangements of large numbers of dislocations.

Recent experimental observations of the cracking behavior of piezoelectric ceramics under combined electrical and mechanical loads contradict predictions made by the existing linear piezoelectric fracture theory. Evidently, a fracture criterion suitable for piezoelectrics must account for material nonlinearity. We identify a region of electrical nonlinearity near the crack tip in which the mechanical response of the material remains linear. For closed-form solutions, we adopt the Dugdale approximation by restricting nonlinear effects to a strip zone ahead of the crack tip. The energy release rate derived using this model agrees qualitatively with empirical measurements of electromechanical fracture load. A remarkable feature of our model is that the energy release rate is strictly independent of the form of the nonlinear electrical constitutive relation. The material may even experience domain switching in the Dugdale zone without affecting the fracture criterion determined by our formulation. We are presently implementing nonlinear finite element algorithms to verify and extend the predictive capability of our analytical models. 
Patterns of both equilibrium and dynamic 2-dimensional arrangements of large numbers of dislocations have been computed numerically. In the case of equilibrium arrangements minimization of the total potential energy of the stressed and dislocated solid is performed; the dynamic studies are accomplished by forward integration of the non-linear stress-dislocation velocity relation. Both types of studies normally would require numerical computations of order $\mathrm{N} 2$, where $\mathrm{N}$ is the total number of dislocations. We have extended the fast-multipole method to treat doubly periodic arrangements of dislocations, so that (1) simulations of large numbers of dislocations are easily accomplished with a method that is $O(N),(2)$ the effects of elastic anisotropy may be included, and (3) the advantage of a fast multipole method with exponentially decaying multipole contributions can be utilized. We are presently studying the kinetic formation of dislocation cell structures and intend to pursue studies or arrangements of 3-dimensional dislocation loops during the coming year.

\section{Stanford University}

Edward L. Ginzton Laboratory

$\$ 231,000$

Stanford, CA $94305-3030$

\section{Optical Techniques for Characterization of High Temperature Superconductors \\ G. Kino}

The aim of this program is to use photothermal techniques to measure the normal carrier density below the transition temperature Tc in hightemperature superconductors, to study the nature of the phase transition and to measure the homogeneity and quality of these materials. A modulated focused laser beam incident on the sample varies its temperature periodically, and a second probe beam a few microns away measures the differential reflectivity (dR/dT) associated with the thermal wave propagating along the sample. Changes in critical temperature in regions less than 100 microns apart have been measured, and the difference in quality of different samples can clearly be seen. Measurement of thermal diffusivity in single YBCO crystals yields good estimates of the variation of normal electron density with temperature. Polarized light observations of single-crystal YBCO near the transition point yield curves as a function of temperature with shapes that are very different depending on the polarization of the probe beam relative to the $A$ and $B$ directions. The nature of the transitions near the critical temperature varies radically with wavelength in the range from 1.3 microns to 0.633 microns.

\section{Stanford University}

Mechanical Engineering Dept

$\$ 235,764$

Stanford, CA 94305

\section{Nonequilibrium Plasma Chemistry}

\section{Kruger, T. Owano}

This research is concerned with optical diagnostics for plasma chemistry and plasma processing, with an emphasis on methods that allow for departures from local thermodynamic equilibrium (such as finite chemical reaction rates, nonequilibrium electron densities and temperatures, and radiation loss effects). Studies in an induction plasma facility show significant nonequilibrium

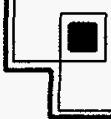


within a downstream reactor, and suggest the possibility of erroneous results when using conventional diagnostics that assume local thermodynamic equilibrium.

Advanced laser-based methods are being developed for measurement of plasma parameters including species concentration and temperature. The primary techniques under investigation are Degenerate Four-Wave Mixing (DFWM) and Cavity Ring-Down Spectroscopy (CRDS). These techniques are being applied to atmospheric pressure and near-atmospheric pressure plasma environments in order to assess the importance of nonequilibrium effects under conditions of interest to plasma chemistry.

Experiments using DFWM to probe $\mathrm{CH}$ and $\mathrm{C}_{2}$ within the reacting plasma have demonstrated the ability to provide sensitive ( $\mathrm{ppm}$ level) detection with submillimeter spatial resolution in the measurement of vibrational temperatures, rotational temperatures, and species concentrations. The use of CRDS to detect $\mathrm{CH}_{3}$ radicals with high sensitivity and high spatial resolution has also been recently demonstrated.

\section{Stanford University}

Dept of Chemistry

Stanford, CA 94305

Topics in a Thermodynamic and Stochastic Theory of Nonlinear Processes Far from Equilibrium

\section{J. Ross}

Research focuses on the thermodynamic and stochastic theory of hydrodynamic processes, such as combinations of chemical reactions, diffusion, thermal conduction, and viscous flow. Such theories have been formulated for each of the individual processes, both linear and nonlinear. Progress has been made exemplified by: a stochastic approach to nonequilibrium chain reactions in disordered systems: Breakdown of eikonal approximation; a study to show that an endogenous calcium oscillator may control early embryonic division; research on deterministic and stochastic asymptotic behavior for nonequilibrium chemical systems with time-dependent rate coefficients; the use of a stochastic potential for a periodically forced nonlinear oscillator; and fluctuation dynamics, thermodynamic analogies and ergodic behavior for nonequilibrium independent rate processes with dynamical disorder; and to show the role of Liapunov functions in the issues of stability and relative stability in reaction diffusion systems with multiple stationary states. This theory has also been extended to study fluctuations near limit cycles in chemical reaction systems. An extensive analysis has been published on the categorization of some oscillatory enzymatic reactions. Prior measurements on glygolytic metabolites by capillary zone electrophoresis have been used for steady-state measurements on the fructose 6-phosphate/fructose 1,6-biophosphate interconverison cycle. 


\section{Stanford University}

Dept of Chemistry

$\$ 95,110$

Stanford, CA 94305

\section{Laser-Based Diagnostics for Plasma Chemistry}

\section{R. Zare}

We have used Cavity Ring-Down Spectroscopy (CRDS) to measure near-infrared overtone transitions of water in atmospheric flames (propane premixed jet and laminar methane-air flat flame burner). CRDS allows more than ten thousand round trips in a high finesse cavity which results in high sensitivity $\left(10^{-8} \mathrm{~cm}^{-1}\right)$ to absorbers in the cell. Moreover, signal output with a welldefined laser beam direction, and the insensitivity of CRDS to a strong emission background present in hostile environments, make CRDS ideal for the study of combustion environments. We have obtained spectra of water vapor from within a flame without significant backgrounds, and have extracted from these spectra the rotational temperature of water vapor as a function of distance from the plane burner surface. These results are in excellent agreement with both simulations and previous experiments reported in the literature.

\section{University Of Texas At Austin}

Center for Studies in Statistical Mech

and Complex Systems

Austin, TX 78712

The Extended Formulation of Dynamics and Computational Tools for Complex Systems I. Prigogine, T. Petrosky, L. Riechl

Over the last year a number of successful applications of our new formulation of dynamics (i.e. our probabilistic extension of dynamics outside the Hilbert space, which incorporates time-symmetry breaking and is irreducible to trajectories or wavefunctions) were obtained. They include the mechanism of the breakdown of integrability of a soluble quantum system (a one-dimensional gas with delta-function interactions) in the thermodynamic limit, as well as kinetic theory of moderately dense classical gas. In the former, it was shown that the nonintegrability invalidates a fundamental principle of quantum mechanics, the "Ritz-Rydberg principle" according to which each frequency has a difference between two energy levels. Indeed it was shown that the eigenvalues of the Liouville-von Neumann operator were not differences of eigenvalues of the Hamiltonian in the thermodynamic limit. Therefore, the basic description for nonintegrable quantum system is in terms of density matrices, and not of wave amplitudes. In the kinetic theory, a long standing problem whether the kinetic equations exist in two dimensional systems was solved. Kinetic equations exist for all dimensions. It was also shown that the nonMarkovian effects, such as long-time tails, invalidate traditional kinetic theory based on a truncation of the BBGKY hierarchy for reduced distribution functions, or on the Green-Kubo formalism based on the decay of fluctuations.

Theoretical studies and numerical simulation of dynamical properties of ballistic electron waveguides has begun. A direct connection between zeros in the electron conductance of these devices and quasibound states related to geometric shape of the waveguides has been found. 


\section{University Of Texas At Austin}

Dept of Physics

Austin, TX 78712

\section{Complex Spatiotemporal Patterns in Nonequilibrium Systems} H. Swinney

The formation of time-dependent spatial patterns is being studied in chemical and physical systems driven far from equilibrium. The goal is to understand what features of behavior are common in diverse pattern-forming systems. What kinds of patterns arise spontaneously as the stress is increased on an initially homogeneous system? What kinds of transitions are permitted between different patterns? What is the role of symmetries in the boundary conditions and the equations of motion? These issues and their relation to applications are being addressed in laboratory experiments, numerical simulations, and analyses on reaction-diffusion systems and vertically vibrated granular media. Experiments on a periodically forced reaction-diffusion system reveal a sequence of resonant regimes where the patterns oscillate at frequencies that are rationally related to the forcing frequency. These frequency-locked regimes are analogous to the frequency-locked tongues of a driven nonlinear pendulum, except that in the spatially expended reactor, different frequencies correspond to different spatial patterns. Vibrated granular media are being studied to determine the dependence of pattern wavelength on forcing frequency. The dispersion relations obtained in experiments and simulations agree well and show two distinct scaling regimes as the drive frequency is varied.

\section{Tufts University}

Department of Mechanical Engineering

$\$ 124,303$

Medford, MA 02155

Solids with Cracks and Pores of Various Shapes: Proper Parameters of Defect Density, Anisotropy and Extraction of Information on Damage from Wavespeed Patterns

\section{Kachanov}

The work is motivated by a number of problems in materials science, geophysics and poroelasticity related to the fact that real materials often contain defects whose shapes are neither spherical nor crack-like, or mixtures of defects of diverse shapes (for example, pores and microcracks). These shape factors strongly affect the effective response and anisotropy; their neglection may lead to wrong interpretations of various experimental data on porous materials.

In particular, they are important for proper interpretation of anisotropic wavespeed patterns in materials with cracks and pores and for the extraction of information on damage from such patterns. Presently, a consistent unified treatment of these issues seems to be lacking. It will be established what kind of information can/cannot be extracted from such a data. For example, preliminary result indicate that the difference between spheres and randomly oriented "needles" in principle cannot be detected by measurements of porosity and longwave speeds, whereas the difference between the spherical and the oblate shapes can be detected.

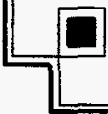




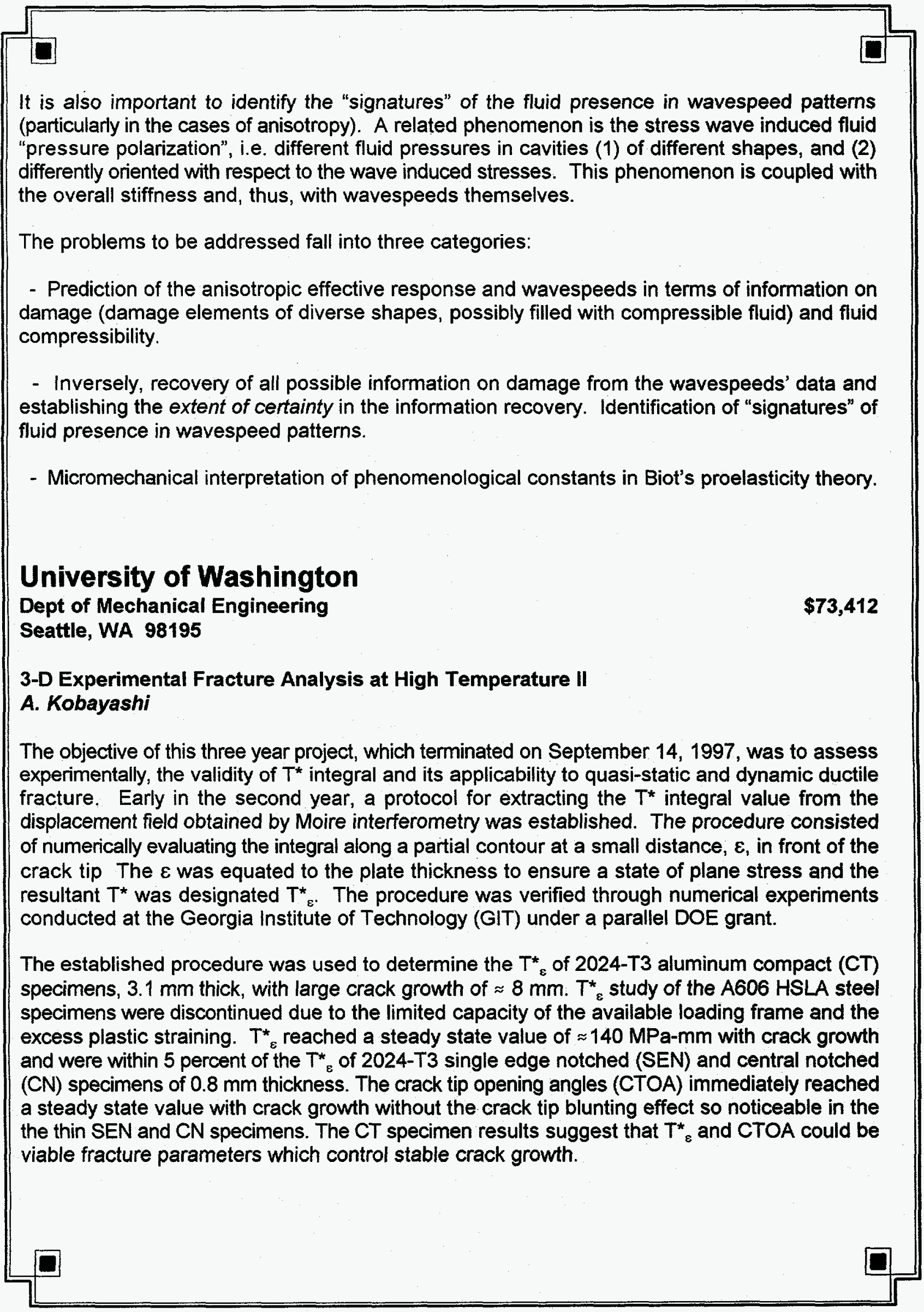

Engineering Research 


\section{Washington University}

Department of Systems Science and Mathematics

$\$ 92,906$

Saint Louis, MO 63130

Visionics: An Integrated Approach to Analysis and Design of Intelligent Machines B. Ghosh

Intelligent systems are systems capable of operating and performing satisfactorily in unknown or partially known environment by learning about the structure and the shape of the ambient where they operate, track the changes on line and adapt to the environment by changing controller structure and goals. A typical motivation for this problem area is autonomous navigation of vehicles and mobile or fixed robots operating in uncertain unknown and time varying environments. For autonomous systems, many diverse subgoals of the mission must in general be considered with the principal goal of the mission is to determine the control actions. Our objective is to study 'Visually Guided Control Problems with specific applications to Vehicle Navigation and Robotics. Primary effort in this proposal is to develop a unified and general set of rational design criteria based on the paradigms of modern systems and control theory. The autonomous guidance system will be adaptable to several kinds of scenarios and problems where intelligent mobile navigation and navigating robots constitute a natural solution. The following three subareas are currently being looked at.

1. Reconstructing 3D Motion and Shape of Moving Objects: This is an ongoing project where spatio-temporal wavelet analysis is being used. The goal here is to obtain filters that are tuned to a suitable orientation and velocity. The filters compute motion and shape parameters locally in parallel.

2. Multisensor Guidance of Robot Manipulators in an Uncalibrated Environment: The goal here is to guide a robot manipulator so as to track and grasp the position and orientation of an object which is dynamically moving in the work-space of the robot. An important challenge here is to recast the manipulation problem from the three dimensional task space to the 'sensor space', that includes cameras and force/torque sensors. 'Sensor fusion' and 'Hybrid Control' are the main focus of this project. Additionally, the problem of tracking a moving part has been considered by introducing a new global 'parallel-tracking' scheme.

3. Autonomous Navigation of Mobile Robots: Goal here is to control a mobile vehicle armed with multiple sensors that can navigate and perform "autonomous path planning", "estimate the shape and motion of target objects" and is capable of tracking in order to keep objects in view.

\section{Washington State University}

Dept of Mech \& Matls Engineering

Pullman, WA 99164-2920

\section{Coupled Particle Dispersion by Three-Dimensional Vortex Structures}

\section{T. Troutt}

The primary objective of this research program is to obtain understanding concerning the role of three-dimensional vortex structures in the dispersion of particles and droplets in free shear flows.

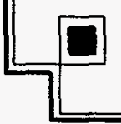

Engineering Research 
This research builds on previous studies which focused on the nature of particle dispersion in large scale quasi two-dimensional vortex structures which are a dominant component of free shear flows. Although three dimensional vortex structures can be quite complex in nature, time scaling quantities such as Stokes number can still be expected to be important for understanding the particle dispersion process.

The study employs time-dependent experimental and numerical techniques to provide information conceming the particulate dispersion process produced by three-dimensional vortex structures. The free shear flows investigated include perturbed plane mixing layers and wakes. Three-dimensional two-phase flow simulation results indicate that streamwise vortex structures can significantly alter the particle dispersion process in plane mixing layers. The influence of these streamwise structures appears to be most pronounced for particles with intermediate value Stokes numbers. The development and nature of these streamwise vortices has also been recently documented experimentally, providing comparison data for evaluating the numerical results.

Additional experimental and numerical studies involving these new observations are presently being pursued. Eventually insights from these three-dimensional particle dispersion results may lead to improvements in the design of practical energy conversion systems.

\section{University Of Wisconsin}

Mechanical Engineering Dept

Milwaukee, Wi 53201

\section{Interfacial Area and Interfacial Transfer in Two-Phase Flow Systems G. Kojasoy}

The objectives of the proposed research program are to develop instrumentation methods, an experimental data base, and an analysis leading to predictive models for describing the interfacial structure and behaviors of horizontal two-phase flows. in terms of the flow structure, the transverse distributions of the local void fraction, interfacial area concentration, fluid particle size and their axial development from the entrance to the exit will be the primary focal point of the research. For the purpose of understanding the dynamic behaviors, the interfacial velocity, wave characteristics, fluid particle coalescence and disintegration will be studied. The axial changes in the distribution of void fraction and interfacial area give the information on the particle coalescence and disintegration. These will be characterized by the collision frequency and interfacial energy and turbulence in the liquid.

A special emphasis will be placed on the further improvement of the multi-sensor resistivity probe method which has been successfully developed and cross-calibrated against other global techniques. The multi-sensor probes will be used together with hot-film probes for the liquid turbulence measurements. These new measurements will give sufficient information to evaluate the local relative velocity and momentum interaction between phases. Final focus of the modeling effort is to develop interfacial area transport equation. which incorporates the mechanistic models for coalescence and disintegration of fluid particles. This transport equation describes dynamical change of the interfacial structure and replaces the conventional model based on flow regime transition criteria.

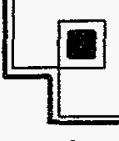


The proposed research program will provide: a) a new scientific instrumentation method for studying detailed interfacial characteristics of two-phase flow, b) benchmark data for the local interfacial area concentration, void fraction distribution, interfacial wave structure, relative velocity and wave propagation velocity for horizontal two-phase flow systems, c) mechanistic models for fluid particle coalescence and disintegration, and d) interfacial area transport equation.

\section{University Of Wisconsin}

Dept of Chemical Engineering Madison, WI 53706

New Process Modeling, Design and Control Strategies for Energy Efficiency, High Product Quality, and Improved Productivity in the Process Industries W. Ray

The process industries are having great difficulty competing in the world market because of high energy costs, high labor rates, and old technology for many processes. This project is concerned with the development of process design and control strategies for improving energy efficiency, product quality, and productivity in the process industry. In particular, (1) the resilient design and control of chemical reactors, and (2) the operation of complex processing systems, will be investigated. Major emphasis in part (1) will be on two important classes of chemical reactors: polymerization processes and packed bed reactors. In part (2), the main focus will be on developing process identification and control procedures which allow the design of advanced control systems based on limited process information and which will work reliably when process parameters change in an unknown manner. Specific topics to be studied include new process identification procedures, nonlinear controller designs, adaptive control methods, and techniques for distributed parameter systems. Both fundamental and immediately applicable results are expected. The theoretical developments are being tested experimentally on pilot scale equipment in the laboratory. These experiments not only allow improvements in theoretical work, but also represent real life demonstrations of the effectiveness of the methods and of the feasibility of implementing them in an industrial environment. The new techniques developed in this project will be incorporated into computer-aided design packages and disseminated to industry. Therefore, it is expected that the work will have an impact on industrial practice. 


\section{Principal Investigators}

Investigator

Page No.

H. Abarbanel

Phone: (619) 534-5590; Fax: (619) 534-7664; e-mail: hdia@hamilton.ucsd.edu . . . . . . . . 7

J. Achenbach

A. Acrivos

G. Ahlers

S. Altobelli

S. Atluri

S. Banerjee

S. Bankoff

J. Barhen

D. Barnett

P. Barton

T. Basar

R. Behringer

B. Berger

P. Bernard

L. Biegler

H. Blanch

I. Blankson

H. Brenner

F. Brust

D. Cannell

A. Caprihan

1. Catton

E.G.D. Cohen

S. Datta

S. Davis

T. Eagar

J. Ekin

J. Epstein

L. Evans

H. Fasel

J. Fincke

L. Freund

E. Fukushima

H. Gao

J. Georgiadis

B. Ghosh

R. Goldstein

Phone: (847) 491-5527; Fax: (847) 491-5227; e-mail: achenbach@nwu.edu . . . . . . . . . . . . 44

Phone: (212) 650-8159; Fax: (212) 650-6835; e-mail: acrivos@scisun.sci.ccny.cuny.edu ..... 41

Phone: (805) 893-3795; Fax: (805) 893-4170; e-mail: guenter@stc.ucsb.edu . . . . . . . . . . 12

Phone: (505) 262-7575; Fax: (505) 262-7043; e-mail: salto@nmr.org . . . . . . . . . . . . 28

Phone: (310) 206-0942; Fax: (310) 267-1922; e-mail: atluri@seas.ucla.edu . . . . . . . . . . 19

Phone: (805) 893-3456; Fax: (805) 893-4731; e-mail: banerjee@anemone.ucsb.edu . . . . . . 12

Phone: (847) 491-5267; Fax: (847) 491-3728; e-mail: gbankoff@casbah.acns.nwu.edu . . . . 44

Phone: (423) 574-7131; Fax: (423) 574-0405; e-mail: barhen@ornl.gov ............. 47

Phone: (650) 723-4143; Fax: (650) 725-4034; e-mail: barnett@leland.stanford.edu . . . . . . . . 56

Phone: (617) 253-6526; Fax: (617) 258-5042; e-mail: pib@mit.edu . . . . . . . . . . . . . 32

Phone: (217) 333-3607; Fax: (217) 244-1653; e-mail: tbasar@isaacs.csl.uiuc.edu ......... 23

Phone: (919) 660-2550; Fax: (919) 660-2525; e-mail: bob@physics.phy.duke.edu . . . . . . . 18

Phone: (301) 405-5283; Fax: (301) 314-9477; e-mail: berger@eng.umd.edu ............ 29

Phone: (301) 405-5272; Fax: (301) 314-9477; e-mail: bernard@eng.umd edu . . . . . . . . . 31

Phone: (412) 268-2232; Fax: (412) 268-7139; e-mail: biegler@cmu edu . . . . . . . . . . . 14

Phone: (510) 642-1387; Fax: (510) 643-1228; e-mail: blanch@socrates berkeley.edu . . . . . . 3

Phone: (216) 433-5823; Fax: (216) 433-3000; e-mail: isaiah.m.blankson@lerc.nasa.gov . . . . 39

Phone: (617) 253-6687; Fax: (617) 258-8224; e-mail: hbrenner@mit.edu . . . . . . . . . . 35

Phone: (614) 424-5034; Fax: (614) 424-3457; e-mail: brust@em.battelle.org . . . . . . . . . . 2

Phone: (805) 893-3150; Fax: (805) 893-4170; e-mail: david@alice.ucsb.edu . . . . . . . . . . 12

Phone: (505) 262-7575; Fax: (505) 262-7043; e-mail: arvind@nmr.org . . . . . . . . . . . . 28

Phone: (310) 825-5320; Fax: (310) 206-4830; e-mail: catton@ ucla.edu . . . . . . . . . . 6

Phone: (212) 327-8855; Fax: (212) 327-8507; e-mail: egdc@rockvax.rockefeller.edu . . . . . . 54

Phone: (303) 492-0287; Fax: (303) 492-3498; e-mail: dattas@spot.colorado.edu . . . . . . . . 15

Phone: (847) 491-5397; Fax: (847) 491-2178; e-mail: sdavis@casbah.acns.nwu.edu . . . . . . 44

Phone: (617) 253-3229; Fax: (617) 252-1773; e-mail: tweagar@mit.edu . . . . . . . . . . . 32

Phone: (303) 497-5448; Fax: (303) 497-5316; e-mail: ekin@boulder.nist.gov . . . . . . . . . . 40

Phone: (208) 526-6874; Fax: (208) 526-0690; e-mail: esj@inel.gov . . . . . . . . . . . . . . 21

Phone: (617) 577-0100; Fax: (617) 577-0722; e-mail: evans@aspentec.com . . . . . . . . . . 32

Phone: (520) 621-2771; Fax: (520) 621-8191; e-mail: faselh@ccit.arizona.edu . . . . . . . . . 1

Phone: (208) 526-2031; Fax: (208) 526-2814; e-mail: jf1@inel.gov . . . . . . . . . . . . . . 19

Phone: (401) 863-1476; Fax: (401) 863-1157; e-mail: freund@isaac.engin.brown.edu ...... 3

Phone: (505) 262-7575; Fax: (505) 262-7043; e-mail: eiichi@nmr.org . . . . . . . . . . . . 28

Phone: (650) 725-2560; Fax. (650) 723-1778; e-mail: gao@am-sun2.stanford.edu . . . . . . . 56

Phone: (217) 244-7578; Fax: (217) 333-1942; e-mail: georgia@staff.uiuc.edu . . . . . . . . . . 18

Phone: (314) 935-6039; Fax: (314) 935-6121; e-mail: ghosh@zach.wustl.edu . . . . . . . . . 62

Phone: (612) 625-5552; Fax: (612) 625-3434; e-mail: rjgumn@mailbox.mail.umn.edu ....... 36

A. Graham Phone: (505) 665-5259; Fax: (505) 665-9521; e-mail: graham@lanl.gov . . . . . . . . . . . 35

C.P. Grigoropoulos Phone: (510) 642-2525; Fax: (510) 642-6163; e-mail: cgrigoro@euler.berkeley.edu . . . . . . 4

I. Grossmann Phone: (412) 268-2230; Fax: (412) 268-7139; e-mail: grossmann@cmu.edu . . . . . . . . . . 14 


\section{Principal Investigators (cont'd - pg. 2)}

H.J.M. Hanley

T. Hanratty

W. Haynes

J. Heberlein

J. Hickman

J. Hunziker

M. Ishii

Y. Iwasa

G. Johnson

J. Johnson

D. Joseph

M. Kachanov

A. Kaufman

R. Kayser

A. Kerstein

G. Kino

A. Kobayashi

G. Kojasoy

R. Kraichnan

E. Krempl

C. Kruger

C. Law

S.C. Li

P. Libby

K. Likharev

$K$. Lindenberg

R. Littlejohn

W. Lloyd

F. McClintock

M. McCready

J. McLaughlin

J. Mayergoyz

C. Menyuk

M. Miksis

1. Minis

L. Mondy

F. Moon

1. Mudawar

S. Namachchivaya

E. Novikov

R.J. Nunge
Phone: (303) 497-3320; Fax: (303) 497-5044; e-mail: hanley@central.bldrdoc.gov . . . . . . . . 40

Phone: (217) 333-1318; Fax: (217) 333-5052; e-mail: thanratt@uiuc.edu . . . . . . . . . . . . . 23

Phone: (303) 497-3247; Fax: (303) 497-5044; e-mail: william. haynes@nist.gov . . . . . . . . . . 41

Phone: (612) 625-4538; Fax: (612) 624-1398; e-mail: jvrh@me.umn.edu . . . . . . . . . . . . . 36

Phone: (202) 994-3863; Fax: (202) 994-5873; e-mail: jhickman@gwu.edu . . . . . . . . . . . . 55

Phone: (202) 334-1571; Fax: (202) 334-2290; e-mail: jhunzike@nae.edu . . . . . . . . . . . . . 37

Phone: (765) 494-4587; Fax: (765) 494-9570; e-mail: grad@ecn.purdue.edu . . . . . . . . . . 50

Phone: (617) 253-5548; Fax: (617) 253-5405; e-mail: iwasa@jokaku.pfc.mit.edu . . . . . . . . 33

Phone: (919) 684-7754; Fax: (919) 684-7122; e-mail: gaj@orion.mc.duke.edu . . . . . . . . . 18

Phone: (208) 526-9021; Fax: (208) 526-0690; e-mail: jsq@inel.gov . . . . . . . . . . . . . . . 20

Phone: (612) 625-0309; Fax: (612) 626-1558; e-mail: joseph@aem.umn.edu . . . . . . . . . . 37

Phone: (617) 627-3318; Fax: (617) 627-3819; e-mail: mkachano@tufts.edu . . . . . . . . . 60

Phone: (510) 486-7899; Fax: (510) 486-4773; e-mail: ankaufman@lbl.gov . . . . . . . . . . 27

Phone: (301) 975-2483; Fax: (301) 869-4020; e-mail: richard.kayser@nist.gov ........... . 41

Phone: (510) 294-2390; Fax: (510) 294-2595; e-mail: kerstein@ca.sandia.gov . . . . . . . . . . 55

Phone: (650) 723-0205; Fax: (650) 725-2533; e-mail: kino@macro.stanford.edu . . . . . . . . . 57

Phone: (206) 543-5488; Fax: (206) 685-8047; e-mail: ask@u.washington edu . . . . . . . . . . . . 61

Phone: (414) 229-5639; Fax: (414) 229-6958; e-mail: kojasoy@csd.uwm.edu . . . . . . . . . . . 63

Phone: (505) 986-3979; Fax: (505) 989-4737; e-mail: rhk@lanl.gov . . . . . . . . . . . . . . . . 26

Phone: (518) 276-6985; Fax: (518) 276-6025; e-mail: krempe@rpi.edu . . . . . . . . . . . . 51

Phone: (650) 723-0977; Fax: (650) 725-1653; e-mail: kruger@soe.stanford.edu . . . . . . . . . . 57

Phone: (609) 258-5271; Fax: (609) 258-6233; e-mail: cklaw@princeton .edu . . . . . . . . . . . . 49

Phone: (619) 534-3168; Fax: (619) 534-6337; e-mail: scli@ames. ucsd.edu . . . . . . . . . . . . . 11

Phone: (619) 534-3168; Fax: (619) 534-5354; e-mail: libby@ames.ucsd.edu . . . . . . . . . . . 11

Phone: (516) 632-8159; Fax: (516) 632-8774; e-mail: klikharev@ccmail.sunysb.edu . . . . . . . 43

Phone: (619) 534-3285; Fax: (619) 534-7244; e-mail: klindenberg@ucsd.edu . . . . . . . . . 8

Phone: (510) 486-7901; Fax: (510) 486-4773; e-mail: rglittlejohn@ibl.gov . . . . . . . . . . . 27

Phone: (208) 526-1509; Fax: (208) 526-0690; e-mail: qrl@inel.gov . . . . . . . . . . . . . . 21

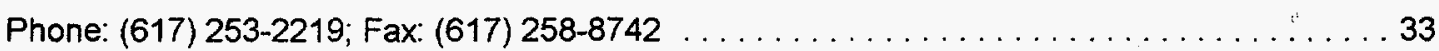

Phone: (219) 631-7146; Fax: (219) 631-8366; e-mail: mccready.1@nd.edu . . . . . . . . . . 47

Phone: (315) 268-6663; Fax: (315) 268-6654; e-mail: jmclau@sun.soe.clarkson edu . . . . . . 15

Phone: (301) 405-3657; Fax: (301) 314-9281; e-mail: isaak@eng.umd.edu ............ . 30

Phone: (410) 455-3501; Fax: (410) 455-6500; e-mail: menyuk@umbc.edu ... . . . . . . . . . 30

Phone: (847) 491-5585: Fax: (847) 491-2178; e-mail: miksis@nwu.edu . . . . . . . . . . . . 45

Phone: (301) 405-5310: Fax: (301) 314-9477; e-mail: minis@eng.umd.edu . . . . . . . . . . . . 29

Phone: (505) 844-1755; Fax: (505) 844-8251; e-mail: lamondy@sandia.gov ............. 35

Phone: (607) 255-7146; Fax: (607) 255-1222; e-mail: fcm3@cornell.edu . . . . . . . . . . . . . . 16

Phone: (765) 494-5705; Fax: (765) 494-0539; e-mail: mudawar@ecn.purdue edu . . . . . . . . 51

Phone: (217) 244-0683; Fax: (217) 244-0720; e-mail: sri@nsgsun.aae.uiuc.edu . . . . . . . . . 24

Phone: (619) 534-0816; Fax: (619) 534-7664; e-mail: enovikov@ucsd.edu . . . . . . . . . . . . 8

Phone: (315) 268-6661; Fax: (315) 268-6654; e-mail: . . . . . . . . . . . . . . . . . . . 15 


\section{Principal Investigators (cont'd - pg. 3)}

E. Oblow
L. Ong
A. Ortega
J. Ottino
T. Owano
L. Parker
D. Parks

T. Petrosky

J. Plawsky

I. Prigogine

A. Prosperetti

V. Protopopescu

S. Putterman

M. Rabinovich

E. Rabinowicz

N.S.V. Rao

W. Ray

D. Reister

W. Reuter

L. Riechl

H. Riecke

I. Rinard

A. Rosakis

J. Ross

T. Rozzell

H. Sehitoglu

G. Settles

J. Sheridan

$R$. Shinnar

G. Sivashinsky

H. Smartt

R. Spanos

G. Stephanopoulos

D. Stoner

C. Surko

H. Swinney

S. Teitel

K. Teischow

C. Tien

S. Torquato

T. Troutt

L. Tsimring
Phone: (423) 574-6187; Fax: (423) 574-7860; e-mail: oblowem@ornl.gov . . . . . . . . . . . . 47

Phone: (301) 405-5343; Fax: (301) 314-9477; e-mail: lawrence@eng.umd.edu . . . . . . . . . . 31

Phone: (520) 621-6787; Fax: (520) 621-8191; e-mail: ortega@ccit. arizona.edu . . . . . . . . . . . 1

Phone: (847) 491-3558; Fax: (847) 491-3728; e-mail: ottino@chem-eng.nwu.edu . . . . . . . . 45

Phone: (650) 723-1295; Fax: (650) 723-1748; e-mail: owano@saha.stanford.edu . . . . . . . . . 57

Phone: (423) 241-4959; Fax: (423) 574-7860; e-mail: parkerle@ornl.gov . . . . . . . . . . . . . 47

Phone: (617) 253-0033; Fax: (617) 258-8742 . . . . . . . . . . . . . . . . . . . . 33

Phone: (512) 471-7253; Fax: (512) 471-9621; e-mail: petrosky@physics.utexas.edu . . . . . . . 59

Phone: (518) 276-6049; Fax: (518) 276-4030; e-mail: plawsky@rpi.edu . . . . . . . . . . . . . . 52

Phone: (512) 471-7253; Fax: (512) 471-9621; e-mail: annie@physics.utexas.edu . . . . . . . . 59

Phone: (410) 516-8534; Fax: (410) 516-7254; e-mail: prosper@titan.me.jhu.edu . . . . . . . . . . 26

Phone: (423) 574-4722; Fax: (423) 574-0405; e-mail: protopopesva@ornl.gov . . . . . . . . . 47

Phone: (310) 825-2269; Fax: (310) 206-5668; e-mail: putterman@physics.ucla.edu . . . . . . . 7

Phone: (619) 534-0816; Fax: (619) 534-7664; e-mail: lev@gibbs.ucsd.edu ... . . . . . . . . . . 9

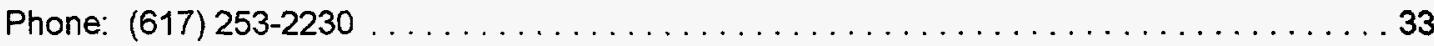

Phone: (423) 574-7517; Fax: (423) 574-7860; e-mail: raons@ornl.gov . . . . . . . . . . . . . . . 47

Phone: (608) 263-4732; Fax: (608) 262-0832; e-mail: ray@engr.wisc.edu . . . . . . . . . . . . . 64

Phone: (423) 574-2272; Fax: (423) 574-7860; e-mail: dbr@ornl.gov . . . . . . . . . . . . . . 47

Phone: (208) 526-1708; Fax: (208) 526-0690; e-mail: wgr2@inel.gov . . . . . . . . . . . . . 21

Phone: (512) 471-7253; Fax: (512) 471-9621; e-mail: reichl@physics. utexas.edu . . . . . . . . 59

Phone: (847) 491-3345; Fax: (847) 491-2178; e-mail: h-riecke@nwu.edu . . . . . . . . . . . 46

Phone: (212) 650-7135; Fax: (212) 650-6686; e-mail: rinard@che-mail.engr.ccny.cuny.edu . . 42

Phone: (818) 395-4523; Fax: (818) 304-0175; e-mail: rosakis@atlantis.caltech.edu ... . . . . 13

Phone: (650) 723-9203; Fax: (650) 723-4817; e-mail: ross@chemistry.stanford.edu .... . . . . 58

Phone: (202) 334-2908; Fax: (202) 334-3419; e-mail: trozzell@nas.edu . . . . . . . . . . . . 38

Phone: (217) 333-4112; Fax: (217) 244-6534; e-mail: huseyin@ux1.cso.uiuc.edu . . . . . . . . . 25

Phone: (814) 863-1504; Fax: (814) 865-0118; e-mail: gss2@psu.edu . . . . . . . . . . . . . 49

Phone: (313) 995-4963; Fax: (313) 995-1150; e-mail: johns@ncms.org . . . . . . . . . . . . . . 39

Phone: (212) 650-6679; Fax: (212) 650-6686; e-mail: shinnar@che-mail.engr.ccny.cuny.edu . . 42

Phone: (212) 650-8157; Fax: (212) 650-6835; e-mail: chemw@ccnyvme .............. 42

Phone: (208) 526-8333; Fax: (208) 526-0690; e-mail: hbs@inel.gov . . . . . . . . . . . . . . 21

Phone: (713) 527-4909; Fax: (713) 285-5191; e-mail: spanos@rice.edu . . . . . . . . . . . . 53

Phone: (617) 253-4583; Fax: (617) 253-3122; e-mail: gregstep@mit.edu . . . . . . . . . . . . . 34

Phone: (208) 526-8786; Fax: (208) 526-0828; e-mail: dls2@inel.gov . . . . . . . . . . . . . . . 20

Phone: (619) 534-6880; Fax: (619) 534-0173; e-mail: csurko@ucsd.edu . . . . . . . . . . . . . 10

Phone: (512) 471-4619; Fax: (512) 471-1558; e-mail: swinney@chaos.ph.utexas.edu . . . . . . 60

Phone: (716) 275-4039; Fax: (716) 275-8527; e-mail: stte@pas.rochester.edu . . . . . . . . . . 53

Phone: (208) 526-1264; Fax: (208) 526-0690; e-mail: telsch@inel.gov . . . . . . . . . . . . . . . . 22

Phone: (510) 643-3886; Fax: (510) 643-3887; e-mail: nancie@uclink4. berkeley.edu . . . . . . . 4

Phone: (609) 258-3341; Fax: (609) 258-2685; e-mail: torquato@matter.princeton.edu . . . . . 50

Phone: (509) 335-4375; Fax: (509) 335-4662; e-mail: troutt@mme.wsu.edu . . . . . . . . . . . 62

Phone: (619) 534-0816; Fax: (619) 534-7664; e-mail: lev@gibbs.ucsd.edu . . . . . . . . . . . . 10 


\section{Principal Investigators (cont'd - pg. 4)}

W.A. Van Der Sluys Phone: (330) 829-7456; Fax: (330) 829-7832; e-mail: alan.w.vandersluys@mcdermott.com . . 29

J. Viñals

Phone: (904) 644-1010; Fax: (904) 644-0098; e-mail vinals@scri.fsu.edu . . . . . . . . . . . . 18

J. Wallace

Phone: (301) 405-5271; Fax: (301) 314-9477; e-mail: wallace@eng.umd.edu

31

G. Wallis

Phone: (603) 646-2789; Fax: (603) 646-3856; e-mail: graham.b.wallis@dartmouth.edu . . . . . 17

Z. Warhaft

Phone: (607) 255-3898; Fax: (607) 255-1222; e-mail: zw16@cornell.edu . . . . . . . . . . 17

P.C. Wayner

Phone: (518) 276-6199; Fax: (518) 276-4030; e-mail: wayner@rpi.edu

52

J.R. Welty

A. Westerberg

Phone: (541) 737-4902; Fax: (541) 737-2600; e-mail: weltyj@ccmail.orst.edu . .

48

G. Wilemski

Phone: (412) 268-2344; Fax: (412) 268-7139; e-mail: a.westerberg@cmu.edu . . . . . . . . . 14

.F. Williams

Phone: (573) 341-4409; Fax: (573) 341-4891; e-mail: wilemski@umr.edu

R. Winston

E. Wolf

Phone: (619) 534-5492; Fax: (619) 534-5354; e-mail: faw@ames.ucsd.edu . . . . . . . . . . 11

Phone: (312) 702-7756; Fax: (312) 702-6317; e-mail: winston@rainbow.uchicago.edu ... . . . 14

I. Wygnanski

Y. Xu

Phone: (716) 275-4397: Fax: (716) 473-0687

54

Phone: (520) 621-6089; Fax: (520) 621-8191; e-mail: wygy@bigdog.engr.arizona.edu ....... 1

R. Zare

Phone: (303) 497-7894; Fax: (303) 497-5316; e-mail: yizixu@boulder.nist.gov . . . . . . . . . . 40

Phone: (650) 723-3062; Fax: (650) 723-9262; e-mail: zare@stanford.edu

.59 